\title{
Somatic Mutations from Whole Exome Sequencing Analysis of the Patients with Biliary Tract Cancer
}

\author{
Kyong-Ah Yoon ${ }^{1}$, Sang Myung Woo ${ }^{2,3 *}$, Yun-Hee Kim ${ }^{3}$, Sun-Young Kong ${ }^{3,4}$, \\ Sung-Sik Han ${ }^{2}$, Sang-Jae Park ${ }^{2}$, Woo Jin Lee ${ }^{2}$ \\ ${ }^{1}$ College of Veterinary Medicine, Konkuk University, Seoul 05029, Korea, ${ }^{2}$ Center for Liver Cancer, National Cancer Center, \\ Goyang 10408, Korea, ${ }^{3}$ Department of Cancer Biomedical Science, National Cancer Center Graduate School of Cancer Science and \\ Policy, Goyang 10408, Korea, ${ }^{4}$ Center for Diagnostic Oncology, National Cancer Center, Goyang 10408, Korea
}

Biliary tract cancer (BTC) is a rare cancer and is associated with a poor prognosis. To understand the genetic characteristics of BTC, we analyzed whole-exome sequencing data and identified somatic mutations in patients with BTC. Tumors and matched blood or normal samples were obtained from seven patients with cholangiocarcinoma who underwent surgical resection. We discovered inactivating mutations of tumor suppressor genes, including APC, TP53, and ARID1A, in three patients. Activating mutations of KRAS and NRAS were also identified. Our analyses identified somatic mutations in Korean patients with BTC.

Keywords: biliary tract neoplasms, cholangiocarcinoma, somatic mutations, whole-exome sequencing

\section{Introduction}

Biliary tract cancer (BTC) is a heterogeneous group of cancers, including gallbladder cancer and extrahepatic and intrahepatic cholangiocarcinoma. BTC has been known as a disease with a poor prognosis, with few treatment options, regardless of its low incidence. In Korea, in 2014, the crude incidence rate of BTC was 11.2 per 100,000 men, and the crude mortality rate was 7.7 per 100,000 in both men and women [1]. Most patients have been diagnosed at an advanced stage. Although surgical resection is a curative treatment, recurrence of disease has been a clinical issue for patients after surgery [2]. Combination treatment with cisplatin plus gemcitabine has been reported as an appropriate treatment for patients with advanced BTC [3].

The genomic characteristics of BTC have been revealed through several studies using high-throughput next-generation sequencing technologies [4-6]. Jiao et al. [5] reported exome sequencing results of 32 patients with intrahepatic cholangiocarcinoma. Inactivating mutations in chromatin remodeling genes, including $B A P 1, A R I D 1 A$, and PBRM1, were identified with frequent mutations in $I D H 1$ and $I D H 2$.
The oncogenes KRAS, PIK3CA, NRAS, GNAS, and ERBB2 were also significantly mutated in BTC [6]. Genomic analyses need to be performed in more BTCs to understand their biology and develop therapeutic strategies.

Here, we reported the whole-exome sequencing (WES) data of seven patients with BTC and analyzed their somatic mutations to compare the genetic features between patients.

\section{Methods}

\section{Patients and collection of clinical data}

All patients had been enrolled in a phase II clinical trial at the National Cancer Center of Korea, as approved by the institutional review board (IRB No. NCCCTC-09-411). Enrolled patients had undergone surgical resection for BTC and were then treated with gemcitabine $\left(1,000 \mathrm{mg} / \mathrm{m}^{2}\right)$ as an adjuvant chemotherapy. The participants voluntarily agreed to donate the materials by signing an informed consent form. Tumor samples were obtained from surgical specimens and blood from seven patients with BTC.

\section{Whole-exome sequencing and data analysis}

We extracted genomic DNA from tissue specimens and 
blood samples using the QIAamp DNA mini kit according to the manufacturer's protocol (Qiagen, Valencia, CA, USA). The concentration and quality of DNA were assessed using a NanoDrop spectrophotometer (Thermo Fisher Scientific, Waltham, MA, USA). WES was performed using the SureSelect Human All Exon V5 Target Enrichment kit (Agilent Technologies, Santa Clara, CA, USA) and processed on the HiSeq 2500 platform (Illumina, San Diego, CA, USA). The size and quantity of the libraries were checked using a 2100 Bioanalyzer (Agilent Technologies).

Sequence reads were aligned to the human reference genome hg19 using the Burrows-Wheeler Aligner-MEM algorithm [7]. The alignments were refined using the Genome Analysis Tool Kit (Broad Institute), and duplicate or low-quality reads were excluded [8]. Somatic mutations were called using Mutect and Strelka with default settings by comparing the sequences of tumor samples with those of matched normal samples $[9,10]$. Only mutations showing a total read depth of greater than 30 in normal samples were considered for further analysis.

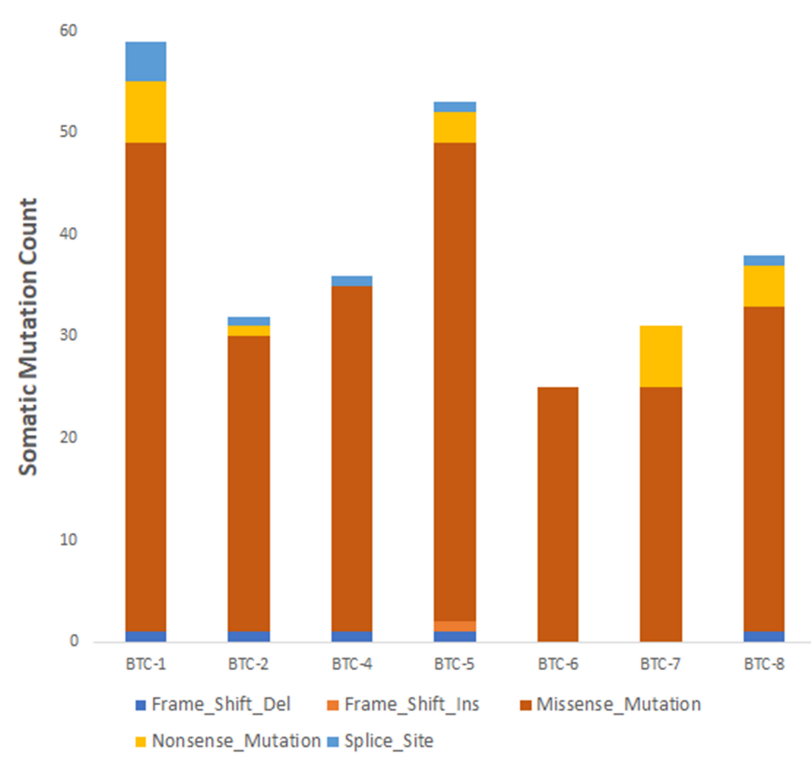

Fig. 1. Somatic mutation count for patients with biliary tract cancer.

Table 1. Demographic information for patients with BTC

\begin{tabular}{clccccc}
\hline Patient ID & Sex & Age $(\mathbf{y})$ & Histologic type & RFS duration (mo) & OS duration (mo) & $\begin{array}{c}\text { Total No. of } \\
\text { somatic mutations }\end{array}$ \\
\hline BTC-1 & Male & 57 & Cholangiocarcinoma & 58.2 & 58.6 & 59 \\
BTC-2 & Female & 70 & Cholangiocarcinoma & 4.1 & 50.1 & 32 \\
BTC-4 & Male & 51 & Cholangiocarcinoma & 1.9 & 6.1 & 36 \\
BTC-5 & Female & 68 & Cholangiocarcinoma & 39.8 & 39.8 & 53 \\
BTC-6 & Female & 69 & Cholangiocarcinoma & 18 & 38.5 & 25 \\
BTC-7 & Male & 55 & Cholangiocarcinoma & 8.4 & 23.3 & 31 \\
BTC-8 & Male & 55 & Cholangiocarcinoma & 8.9 & 15.8 & 38 \\
\hline
\end{tabular}

BTC, biliary tract cancer; RFS, recurrence-free survival; OS, overall survival.

Table 2. Somatic mutations that were annotated with cancer gene census

\begin{tabular}{|c|c|c|c|c|}
\hline Hugo_Symbol & Sample ID & Variant classification & cDNA change & Protein change \\
\hline$A P C$ & BTC-1 & Nonsense & c. $4660 \mathrm{G}>\mathrm{T}$ & p.E1554* \\
\hline$A P C$ & BTC-8 & Frameshift & c.4661delA & p.E1554fs \\
\hline$A R I D 1 A$ & BTC-4 & Frameshift & c.937delG & p.G314fs \\
\hline DNMT3A & BTC-7 & Missense & c. $946 \mathrm{~A}>\mathrm{G}$ & p.I316V \\
\hline$E L N$ & BTC-4 & Missense & c.593C $>\mathrm{T}$ & p.P198L \\
\hline HIP1 & BTC-4 & Missense & c. $1300 C>T$ & p.R434W \\
\hline KDM5A & BTC-6 & Missense & c. $2791 \mathrm{G}>\mathrm{A}$ & p.G931R \\
\hline KIAA1549 & BTC-2 & Missense & c. $508 \mathrm{C}>\mathrm{T}$ & p.R170W \\
\hline KRAS & BTC-7 & Missense & c. $35 \mathrm{G}>\mathrm{T}$ & p.G12V \\
\hline KRAS & BTC-8 & Missense & c. $38 \mathrm{G}>\mathrm{A}$ & p.G13D \\
\hline$L P P$ & BTC-7 & Missense & c. $457 \mathrm{C}>\mathrm{T}$ & p.P153S \\
\hline NRAS & BTC-5 & Missense & c. $182 A>G$ & p.Q61R \\
\hline NTRK1 & BTC-7 & Missense & c. $2297 \mathrm{G}>\mathrm{A}$ & p.R766Q \\
\hline$R B 1$ & BTC-6 & Missense & c. $2402 \mathrm{G}>\mathrm{C}$ & p.G801A \\
\hline RBM15 & BTC-1 & Missense & c. $1519 \mathrm{~T}>\mathrm{G}$ & p.W507G \\
\hline TP53 & BTC-8 & Nonsense & c. $1024 \mathrm{C}>\mathrm{T}$ & p.R342* \\
\hline
\end{tabular}




\section{Results and Discussion}

The demographic and clinical characteristics of the patients with BTC, including age, sex, cancer stage, recurrence, and survival, are summarized in Table 1.

The patients included three females and four males with an average age of 60 years. Among the seven patients, five patients were defined as experiencing recurrence of disease after surgery.

We analyzed whole-exome sequencing data of matched tumor and normal DNA from the patients. The mean coverage depth for all samples is demonstrated in Supplementary Table 1. In total, 274 somatic mutations in protein-coding regions were called by both callers, Strelka and Mutect. The average number of somatic mutations per case was 34, showing a range of 25 to 59 (Fig. 1). We found two patients harboring activating mutations in KRAS, such as G12V and G13D (Table 2). Missense mutations in codon 61 of the NRAS gene and codon 766 of the NTRK1 gene were also detected in two patients. A nonsense mutation in TP53 was found in a patient, and APC was inactivated by frameshift mutations in two patients. A patient harbored a frameshift mutation that was caused by a deletion of G in the ARID1A gene. Detailed information on the somatic mutations is provided in Supplementary Table 2.

This study analyzed somatic mutations using WES in a limited number of patients with BTC. To understand the genetic features of BTC, more data need to be analyzed from more patients.

ORCID: Kyong-Ah Yoon: https://orcid.org/0000-0002-98237393; Sang Myung Woo: https://orcid.org/0000-0003-37864403; Yun-Hee Kim: https://orcid.org/0000-0001-7571-9958; Sun-Young Kong: https://orcid.org/0000-0003-0620-4058; Sung-Sik Han: https://orcid.org/0000-0001-7047-7961; Sang-Jae Park: https://orcid.org/0000-0001-5582-9420; Woo Jin Lee: https://orcid.org/0000-0003-2115-6701

\section{Authors' contribution}

Conceptualization: KAY, SMW, WJL

Data curation: KAY, SMW

Formal analysis: KAY, SMW, YHK, SYK, WJL

Funding acquisition: SMW, WJL

Investigation: KAY, SMW, SSH, SJP, WJL

Methodology: KAY, SMW, YHK, SYK

Project administration: KAY, SMW, WJL

Software: KAY

Supervision: KAY, SMW, SSH, SJP, WJL

Validation: KAY, SMW, YHK, SYK, WJL

Visualization: K.-A,Y., S.M.W.

Writing - original draft: K.-A,Y., S.M.W
Writing - review and editing: KAY, SMW, YHK, SYK, SSH, SJP, WJL

\section{Conflicts of Interest}

No potential conflicts of interest relevant to this article was reported.

\section{Acknowledgments}

This work was supported by a National Cancer Center Research Grant 1510690 to W.G. Lee.

\section{Supplementary material}

Supplementary data including two tables can be found with this article online at https://doi.org/10.5808/GI.2018. 16.4.e35.

\section{References}

1. Jung KW, Won YJ, Oh CM, Kong HJ, Lee DH, Lee KH, et al. Cancer statistics in Korea: incidence, mortality,survival, and prevalence in 2014. Cancer Res Treat 2017;49:292-305.

2. Jung SJ, Woo SM, Park HK, Lee WJ, Han MA, Han SS, et al. Patterns of initial disease recurrence after resection of biliary tract cancer. Oncology 2012;83:83-90.

3. Valle J, Wasan H, Palmer DH, Cunningham D, Anthoney A, Maraveyas A, et al. Cisplatin plus gemcitabine versus gemcitabine for biliary tract cancer. N Engl J Med 2010;362:1273-1281.

4. Ahn DH, Javle M, Ahn CW, Jain A, Mikhail S, Noonan AM, et al. Next-generation sequencing survey of biliary tract cancer reveals the association between tumor somatic variants and chemotherapy resistance. Cancer 2016;122:3657-3666.

5. Jiao Y, Pawlik TM, Anders RA, Selaru FM, Streppel MM, Lucas $\mathrm{DJ}$, et al. Exome sequencing identifies frequent inactivating mutations in BAP1, ARID1A and PBRM1 in intrahepatic cholangiocarcinomas. Nat Genet 2013;45:1470-1473.

6. Nakamura H, Arai Y, Totoki Y, Shirota T, Elzawahry A, Kato M, et al. Genomic spectra of biliary tract cancer. Nat Genet 2015;47:1003-1010.

7. Li H, Durbin R. Fast and accurate short read alignment with Burrows-Wheeler transform. Bioinformatics 2009;25:1754-1760.

8. McKenna A, Hanna M, Banks E, Sivachenko A, Cibulskis K, Kernytsky A, et al. The Genome Analysis Toolkit: a MapReduce framework for analyzing next-generation DNA sequencing data. Genome Res 2010;20:1297-1303.

9. Cibulskis K, Lawrence MS, Carter SL, Sivachenko A, Jaffe D, Sougnez $\mathrm{C}$, et al. Sensitive detection of somatic point mutations in impure and heterogeneous cancer samples. Nat Biotechnol 2013;31:213-219.

10. Saunders CT, Wong WS, Swamy S, Becq J, Murray LJ, Cheetham RK. Strelka: accurate somatic small-variant calling from sequenced tumor-normal sample pairs. Bioinformatics 2012;28:1811-1817. 


\section{SUPPLEMENTARY INFORMATION}

\section{Somatic Mutations from Whole Exome Sequencing Analysis of the Patients with Biliary Tract Cancer}

\section{Kyong-Ah Yoon ${ }^{1}$, Sang Myung Woo ${ }^{2,3 *}$, Yun-Hee $\mathrm{Kim}^{3}$, Sun-Young Kong ${ }^{3,4}$, Sung-Sik Han', Sang-Jae Park, Woo Jin Lee}

${ }^{1}$ College of Veterinary Medicine, Konkuk University, Seoul 05029, Korea, ${ }^{2}$ Center for Liver Cancer, National Cancer Center, Goyang 10408, Korea, ${ }^{3}$ Department of Cancer Biomedical Science, National Cancer Center Graduate School of Cancer Science and Policy, Goyang 10408, Korea, ${ }^{4}$ Center for Diagnostic Oncology,

National Cancer Center, Goyang 10408, Korea 


\section{Supplementary Table 1. Coverage Information}

\begin{tabular}{|c|c|c|c|c|c|c|c|c|c|c|}
\hline Sample & $\mathrm{X} 1$ & $\times 5$ & X10 & $\mathrm{X} 15$ & X20 & $\mathrm{X} 25$ & X30 & $\times 50$ & $\begin{array}{l}\text { Total bases } \\
\text { aligned(bases) }\end{array}$ & $\begin{array}{l}\text { Mean coverage } \\
\text { depth }\end{array}$ \\
\hline BTC_1N & $99.96 \%$ & $99.77 \%$ & $99.22 \%$ & $98.14 \%$ & $96.33 \%$ & $93.69 \%$ & $90.19 \%$ & $69.94 \%$ & $6,034,974,927$ & 80.99 \\
\hline BTC_1T & $99.97 \%$ & $99.90 \%$ & $99.71 \%$ & $99.38 \%$ & $98.84 \%$ & $98.03 \%$ & $96.92 \%$ & $89.02 \%$ & $9,863,779,665$ & 132.38 \\
\hline BTC_2N & $99.86 \%$ & $99.66 \%$ & $99.15 \%$ & $98.17 \%$ & $96.48 \%$ & $93.95 \%$ & $90.45 \%$ & $69.62 \%$ & $5,978,637,815$ & 80.24 \\
\hline BTC_2T & $99.88 \%$ & $99.79 \%$ & $99.63 \%$ & $99.38 \%$ & $99.00 \%$ & $98.46 \%$ & $97.71 \%$ & $92.10 \%$ & $11,086,728,430$ & 148.79 \\
\hline BTC_4N & $99.97 \%$ & $99.80 \%$ & $99.30 \%$ & $98.32 \%$ & $96.67 \%$ & $94.26 \%$ & $91.07 \%$ & $72.14 \%$ & $6,345,622,329$ & 85.16 \\
\hline BTC_4T & $99.98 \%$ & $99.90 \%$ & $99.72 \%$ & $99.43 \%$ & $98.98 \%$ & $98.33 \%$ & $97.44 \%$ & $91.14 \%$ & $10,760,737,151$ & 144.41 \\
\hline BTC_5N & $99.86 \%$ & $99.62 \%$ & $98.95 \%$ & $97.66 \%$ & $95.53 \%$ & $92.44 \%$ & $88.40 \%$ & $66.57 \%$ & $5,823,253,443$ & 78.15 \\
\hline BTC_5T & $99.87 \%$ & $99.78 \%$ & $99.57 \%$ & $99.21 \%$ & $98.65 \%$ & $97.88 \%$ & $96.83 \%$ & $89.50 \%$ & $10,599,020,827$ & 142.24 \\
\hline BTC_6N & $99.87 \%$ & $99.67 \%$ & $99.16 \%$ & $98.14 \%$ & $96.43 \%$ & $93.80 \%$ & $90.22 \%$ & $69.15 \%$ & $5,968,127,744$ & 80.09 \\
\hline BTC_6T & $99.88 \%$ & $99.79 \%$ & $99.63 \%$ & $99.37 \%$ & $98.99 \%$ & $98.43 \%$ & $97.66 \%$ & $91.94 \%$ & $10,923,830,027$ & 146.60 \\
\hline BTC_7N & $99.97 \%$ & $99.78 \%$ & $99.25 \%$ & $98.21 \%$ & $96.48 \%$ & $93.96 \%$ & $90.63 \%$ & $71.11 \%$ & $6,186,543,991$ & 83.03 \\
\hline BTC_7T & $99.98 \%$ & $99.88 \%$ & $99.66 \%$ & $99.27 \%$ & $98.66 \%$ & $97.75 \%$ & $96.48 \%$ & $87.79 \%$ & $9,956,458,889$ & 133.62 \\
\hline BTC_8N & $99.97 \%$ & $99.76 \%$ & $99.17 \%$ & $97.97 \%$ & $95.96 \%$ & $93.09 \%$ & $89.35 \%$ & $68.81 \%$ & $5,998,051,912$ & 80.50 \\
\hline BTC_8T & $99.98 \%$ & $99.89 \%$ & $99.68 \%$ & $99.34 \%$ & $98.82 \%$ & $98.06 \%$ & $97.01 \%$ & $89.49 \%$ & $10,131,605,777$ & 135.97 \\
\hline
\end{tabular}




\section{Supplementary Table 2. Detailed information on total somatic mutations}

\begin{tabular}{|c|c|c|c|c|c|c|c|c|c|c|c|}
\hline Patient ID & Hugo_Sym Er & ntrez_Gen Ch & noso & art_position & End_position & Variant_Classification & Reference_ & -Tumor_Sec & q cDNA_Change & Codon_ChiProtein_Cr & hescription \\
\hline BTC-1 & APC & 324 & 5 & 112174759 & 112174760 & Frame_Shift_Del & AG & - & c.3468_3469delA & C.c.(3466-34 p.EE1156fs & s adenomatous polyposis coli \\
\hline BTC-1 & KRTAP19- $\epsilon$ & 337973 & 21 & 31914024 & 31914024 & Nonsense_Mutation & G & $\mathrm{T}$ & c. $129 C>A$ & c.(127-129 p.C43* & keratin associated protein $19-6$ \\
\hline BTC-1 & PABPC4L & 132430 & 4 & 135121319 & 135121319 & Nonsense_Mutation & G & A & $\mathrm{c} .856 \mathrm{C}>\mathrm{T}$ & c. $(856-858$ p.R286* & poly(A) binding protein, cytoplasmic 4-like \\
\hline BTC-1 & APC & 324 & 5 & 112175951 & 112175951 & Nonsense_Mutation & G & $\mathrm{T}$ & $c .4660 G>T$ & c. $(4660-46$ p.E1554* & adenomatous polyposis coli \\
\hline BTC-1 & C6orf222 & 389384 & 6 & 36290159 & 36290159 & Nonsense_Mutation & G & c & c. $1532 C>G$ & c.(1531-15 p.S511* & chromosome 6 open reading frame 222 \\
\hline BTC-1 & $\mathrm{ABCA} 1$ & 19 & 9 & 107553269 & 107553269 & Nonsense_Mutation & G & C & c. $5861 C>G$ & c.(5860-58 p.S1954* & ATP-binding cassette, sub-family $A(A B C 1)$, member 1 \\
\hline BTC-1 & AMER1 & $139285 X$ & & 63412530 & 63412530 & Nonsense_Mutation & G & A & c. $637 \mathrm{C}>\mathrm{T}$ & c.(637-639 p.Q213* & APC membrane recruitment protein 1 \\
\hline BTC-1 & MFAP2 & 4237 & 1 & 17302995 & 17302995 & Splice_Site & $\mathrm{C}$ & A & & c.e5+1 & microfibrillar-associated protein 2 \\
\hline BTC-1 & HIF3A & 64344 & 19 & 46825222 & 46825222 & Splice_Site & C & $\mathrm{T}$ & c. $1334 C>T$ & c.(1333-13 p.S445L & hypoxia inducible factor 3 , alpha subunit \\
\hline BTC-1 & MAP3K13 & 9175 & 3 & 185155234 & 185155234 & Splice_Site & G & C & & c.e3-1 & mitogen-activated protein kinase kinase kinase 13 \\
\hline BTC-1 & DAB2IP & 153090 & 9 & 124329507 & 124329507 & Splice_Site & G & A & c. $40 \mathrm{G}>\mathrm{A}$ & c.(40-42)Gip.E14K & DAB2 interacting protein \\
\hline BTC-1 & $\mathrm{ABCC} 2$ & 1244 & 10 & 101591752 & 101591752 & Missense_Mutation & C & T & c. $3122 C>T$ & c.(3121-31 p.A1041V & ATP-binding cassette, sub-family C (CFTR/MRP), member 2 \\
\hline BTC-1 & ACE & 1636 & 17 & 61570945 & 61570945 & Missense_Mutation & G & A & c. $1339 \mathrm{G}>\mathrm{A}$ & c.(1339-13.p.V447M & angiotensin I converting enzyme \\
\hline BTC-1 & ACTN2 & 88 & 1 & 236899007 & 236899007 & Missense_Mutation & C & T & c. $770 \mathrm{C}>\mathrm{T}$ & c.(769-771 p.A257V & actinin, alpha 2 \\
\hline BTC-1 & ACTN4 & 81 & 19 & 39207832 & 39207832 & Missense_Mutation & G & A & c. $1019 \mathrm{G}>\mathrm{A}$ & c.(1018-10 p.R340H & actinin, alpha 4 \\
\hline BTC-1 & ACVR1B & 91 & 12 & 52379016 & 52379016 & Missense_Mutation & C & A & c. $864 C>A$ & c.(862-864 p.N288K & activin $A$ receptor, type IB \\
\hline BTC-1 & ADCY5 & 111 & 3 & 123166420 & 123166420 & Missense_Mutation & $\mathrm{C}$ & T & c. $973 \mathrm{G}>\mathrm{A}$ & c.(973-975 p.A325T & adenylate cyclase 5 \\
\hline BTC-1 & ADCY8 & 114 & 8 & 131949376 & 131949376 & Missense_Mutation & C & $\mathrm{T}$ & c. $1424 G>A$ & c.(1423-14 p.R475H & adenylate cyclase 8 (brain) \\
\hline BTC-1 & ANO3 & 63982 & 11 & 26463533 & 26463533 & Missense_Mutation & $\mathrm{T}$ & $\mathrm{C}$ & C. $115 T>C$ & c. $(115-117$ p.C39R & anoctamin 3 \\
\hline BTC-1 & APC2 & 10297 & 19 & 1457029 & 1457029 & Missense_Mutation & G & A & c. $994 \mathrm{G}>\mathrm{A}$ & c.(994-996 p.A332T & adenomatosis polyposis coli 2 \\
\hline BTC-1 & AQPEP & 0 & 5 & 115298621 & 115298621 & Missense_Mutation & G & A & c. $307 \mathrm{G}>\mathrm{A}$ & c.(307-309 p.V103M & \\
\hline BTC-1 & AUTS2 & 26053 & 7 & 69364311 & 69364311 & Missense_Mutation & $\mathrm{C}$ & T & c. $349 C>T$ & c.(349-351 p.R117C & autism susceptibility candidate 2 \\
\hline BTC-1 & C11orf63 & 79864 & 11 & 122775018 & 122775018 & Missense_Mutation & C & $\mathrm{T}$ & c.730C $>\mathrm{T}$ & c.(730-732 p.R244C & chromosome 11 open reading frame 63 \\
\hline BTC-1 & C5orf42 & 65250 & 5 & 37153858 & 37153858 & Missense_Mutation & $\mathrm{T}$ & A & c. $8195 \mathrm{~A}>\mathrm{T}$ & c.(8194-81 p.D2732V & chromosome 5 open reading frame 42 \\
\hline BTC-1 & $\mathrm{CDH} 2$ & 1000 & 18 & 25572711 & 25572711 & Missense_Mutation & C & $\mathrm{T}$ & c. $1159 \mathrm{G}>\mathrm{A}$ & c.(1159-11 p.A387T & cadherin 2 , type $1, \mathrm{~N}$-cadherin (neuronal) \\
\hline BTC-1 & CHST9 & 83539 & 18 & 24628462 & 24628462 & Missense_Mutation & $\mathrm{C}$ & $\mathrm{T}$ & c. $127 \mathrm{G}>\mathrm{A}$ & c. $(127-129$ p.V43M & carbohydrate ( $\mathrm{N}$-acetylgalactosamine 4-0) sulfotransferase 9 \\
\hline BTC-1 & CYP11B1 & 1584 & 8 & 143956558 & 143956558 & Missense_Mutation & C & T & c. $1426 \mathrm{G}>\mathrm{A}$ & c.(1426-14 p.V476M & cytochrome P450, family 11 , subfamily B, polypeptide 1 \\
\hline BTC-1 & DNAH10 & 196385 & 12 & 124341718 & 124341718 & Missense_Mutation & G & A & c. $6200 \mathrm{G}>\mathrm{A}$ & c. $(6199-62$ p.R2067H & dynein, axonemal, heavy chain 10 \\
\hline BTC-1 & DSCAM & 1826 & 21 & 41559851 & 41559851 & Missense_Mutation & $\mathrm{C}$ & $\mathrm{T}$ & c. $2617 G>A$ & c.(2617-26 p.E873K & Down syndrome cell adhesion molecule \\
\hline BTC-1 & ELF3 & 1999 & 1 & 201983140 & 201983140 & Missense_Mutation & G & A & c. $989 \mathrm{G}>\mathrm{A}$ & c.(988-990 p.S330N & $\begin{array}{l}\text { E74-like factor } 3 \text { (ets domain transcription factor, epithelial- } \\
\text { specific ) }\end{array}$ \\
\hline BTC-1 & FGD3 & 89846 & 9 & 95738639 & 95738639 & Missense_Mutation & C & T & c. $101 C>T$ & c. $(100-102$ p.A34V & FYVE, RhoGEF and PH domain containing 3 \\
\hline BTC-1 & FUBP3 & 8939 & 9 & 133493210 & 133493210 & Missense_Mutation & G & A & c. $594 \mathrm{G}>\mathrm{A}$ & c.(592-594 p.M198I & far upstream element (FUSE) binding protein 3 \\
\hline BTC-1 & HAND1 & 9421 & 5 & 153855452 & 153855452 & Missense_Mutation & G & T & c. $562 C>A$ & c. (562-564 p.P188T & heart and neural crest derivatives expressed 1 \\
\hline BTC-1 & KCNS2 & 3788 & 8 & 99440299 & 99440299 & Missense_Mutation & G & A & c. $92 \mathrm{G}>\mathrm{A}$ & c. $(91-93) c(p . R 31 \mathrm{H}$ & $\begin{array}{l}\text { potassium voltage-gated channel, delayed-rectifier, subfamily } S \text {, } \\
\text { member } 2\end{array}$ \\
\hline BTC-1 & KIAA1429 & 25962 & 8 & 95503865 & 95503865 & Missense_Mutation & $\mathrm{C}$ & G & c. $5081 G>C$ & c.(5080-50 p.R1694T & KIAA1429 \\
\hline BTC-1 & KRTAP19-1 & 337882 & 21 & 31852374 & 31852374 & Missense_Mutation & $\mathrm{C}$ & T & c. $263 \mathrm{G}>\mathrm{A}$ & c. $(262-264$ p.G88D & keratin associated protein $19-1$ \\
\hline BTC-1 & LRP1B & 53353 & 2 & 141004693 & 141004693 & Missense_Mutation & G & T & c. $13286 C>A$ & c.(13285-1 p.P4429Q & low density lipoprotein receptor-related protein 1B \\
\hline BTC-1 & LRRK2 & 120892 & 12 & 40689336 & 40689336 & Missense_Mutation & G & $\mathrm{T}$ & c. $2986 G>T$ & c.(2986-29 p.D996Y & leucine-rich repeat kinase 2 \\
\hline BTC-1 & MAP2K1 & 5604 & 15 & 66727455 & 66727455 & Missense_Mutation & G & C & c. $171 \mathrm{G}>\mathrm{C}$ & c. $(169-171$ p.K57N & mitogen-activated protein kinase kinase 1 \\
\hline
\end{tabular}




\begin{tabular}{|c|c|c|c|c|c|c|c|c|c|c|}
\hline BTC-1 & MBTD1 & 54799 & 17 & 49296318 & 49296318 Missense_Mutation & $A$ & $C$ & c. $376 \mathrm{~T}>\mathrm{G}$ & c.(376-378 p.L126V & mbt domain containing 1 \\
\hline BTC-1 & MUC19 & 283463 & 12 & 40820354 & 40820354 Missense_Mutation & G & A & c. $332 \mathrm{G}>\mathrm{A}$ & c.(331-333 p.R111H & mucin 19 , oligomeric \\
\hline BTC-1 & MYLK2 & 85366 & 20 & 30419645 & 30419645 Missense_Mutation & $\mathrm{G}$ & A & c. $1564 G>A$ & c.(1564-15 p.V522I & myosin light chain kinase 2 \\
\hline BTC-1 & PCDH17 & 27253 & 13 & 58207860 & 58207860 Missense_Mutation & $\mathrm{G}$ & A & c. $1180 G>A$ & c.(1180-11 p.G394R & protocadherin 17 \\
\hline BTC-1 & PLIN4 & 729359 & 19 & 4510530 & 4510530 Missense_Mutation & $\mathrm{G}$ & A & c. $3400 C>T$ & c.(3400-34 p.R1134C & perilipin 4 \\
\hline BTC-1 & PRRC2B & 84726 & 9 & 134322013 & 134322013 Missense_Mutation & $A$ & G & $c .839 A>G$ & c.(838-840 p.D280G & proline-rich coiled-coil 2B \\
\hline BTC-1 & RBM15 & 64783 & 1 & 110883546 & 110883546 Missense_Mutation & $\mathrm{T}$ & G & c.1519T>G & c.(1519-15 p.W507G & RNA binding motif protein 15 \\
\hline BTC-1 & RP1-139D & 0 & 6 & 42123292 & 42123292 Missense_Mutation & $\mathrm{G}$ & T & c. $14 \mathrm{G}>\mathrm{T}$ & c. $(13-15) a(p . S 5 \mid$ & \\
\hline BTC-1 & SDK1 & 221935 & 7 & 4273010 & 4273010 Missense_Mutation & $\mathrm{C}$ & $\mathrm{T}$ & c. $5891 C>T$ & c.(5890-58 p.A1964V & sidekick cell adhesion molecule 1 \\
\hline BTC-1 & SPRY2 & 10253 & 13 & 80911584 & 80911584 Missense_Mutation & $\mathrm{G}$ & C & c. $257 C>G$ & c.(256-258 p.P86R & sprouty homolog 2 (Drosophila) \\
\hline BTC-1 & ST6GALNA & 81849 & 1 & 77509964 & 77509964 Missense_Mutation & $C$ & A & c. $337 C>A$ & c.(337-339 p.Q113K & $\begin{array}{l}\text { ST6 (alpha- } \mathrm{N} \text {-acetyl-neuraminyl-2,3-beta-galactosyl-1,3)- } \mathrm{N} \text { - } \\
\text { acetylgalactosaminide alpha-2,6-sialyltransferase } 5\end{array}$ \\
\hline BTC-1 & SYT3 & 84258 & 19 & 51135883 & 51135883 Missense_Mutation & $\mathrm{C}$ & $\mathrm{T}$ & c. $334 \mathrm{G}>\mathrm{A}$ & c.(334-336 p.G112R & synaptotagmin III \\
\hline BTC-1 & TADA2A & 6871 & 17 & 35837062 & 35837062 Missense_Mutation & G & C & c. $1307 \mathrm{G}>\mathrm{C}$ & c.(1306-13 p.R436T & transcriptional adaptor $2 \mathrm{~A}$ \\
\hline BTC-1 & TGM2 & 7052 & 20 & 36793594 & 36793594 Missense_Mutation & $\mathrm{C}$ & $\mathrm{T}$ & $c .7 \mathrm{G}>\mathrm{A}$ & c. (7-9)Gag p.E3K & transglutaminase 2 \\
\hline BTC-1 & TMEM184I & 25829 & 22 & 38643871 & 38643871 Missense_Mutation & $\mathrm{T}$ & C & c. $97 \mathrm{~A}>\mathrm{G}$ & c.(97-99)Аср.ТЗ3А & transmembrane protein $184 \mathrm{~B}$ \\
\hline BTC-1 & TRPC1 & 7220 & 3 & 142499703 & 142499703 Missense_Mutation & A & $\mathrm{T}$ & c.792A $>\mathrm{T}$ & c.(790-792 p.Q264H & transient receptor potential cation channel, subfamily $C$, member \\
\hline BTC-1 & TSHZ3 & 57616 & 19 & 31770589 & 31770589 Missense_Mutation & G & C & c. $110 C>G$ & c.(109-111 p.A37G & teashirt zinc finger homeobox 3 \\
\hline BTC-1 & TSPAN14 & 81619 & 10 & 82271948 & 82271948 Missense_Mutation & $\mathrm{T}$ & G & c.499T > G & c.(499-501 p.Y167D & tetraspanin 14 \\
\hline BTC-1 & ZFR & 51663 & 5 & 32395347 & 32395347 Missense_Mutation & C & $\mathrm{T}$ & c. $1897 \mathrm{G}>\mathrm{A}$ & c.(1897-18 p.E633K & zinc finger RNA binding protein \\
\hline BTC-1 & ZFYVE26 & 23503 & 14 & 68264958 & 68264958 Missense_Mutation & $C$ & A & c. $2021 \mathrm{G}>\mathrm{T}$ & c.(2020-20 p.G674V & zinc finger, FYVE domain containing 26 \\
\hline BTC-2 & EPHA2 & 1969 & 1 & 16464447 & 16464447 Frame_Shift_Del & G & - & c.1213delC & c.(1213-12 p.H405fs & EPH receptor A2 \\
\hline BTC-2 & LGI4 & 163175 & 19 & 35617610 & 35617610 Nonsense_Mutation & $\mathrm{C}$ & $\mathrm{T}$ & $c .863 \mathrm{G}>\mathrm{A}$ & c.(862-864 p.W288* & leucine-rich repeat LGI family, member 4 \\
\hline BTC-2 & EPHA2 & 1969 & 1 & 16456720 & 16456720 Splice_Site & $C$ & $\mathrm{~T}$ & & c.e15+1 & $\mathrm{EPH}$ receptor $\mathrm{A} 2$ \\
\hline BTC-2 & C17orf97 & 400566 & 17 & 260315 & 260315 Missense_Mutation & G & $\mathrm{T}$ & c. $182 \mathrm{G}>\mathrm{T}$ & c.(181-183 p.G61V & chromosome 17 open reading frame 97 \\
\hline BTC-2 & CAMK2N1 & 55450 & 1 & 20810156 & 20810156 Missense_Mutation & G & A & c. $223 C>T$ & c.(223-225 p.P75S & calcium/calmodulin-dependent protein kinase II inhibitor 1 \\
\hline BTC-2 & CNBD1 & 168975 & 8 & 88249206 & 88249206 Missense_Mutation & G & A & c. $637 \mathrm{G}>\mathrm{A}$ & c. (637-639 p.V213M & cyclic nucleotide binding domain containing 1 \\
\hline BTC-2 & COL14A1 & 7373 & 8 & 121292323 & 121292323 Missense_Mutation & $\mathrm{G}$ & A & c. $3631 \mathrm{G}>\mathrm{A}$ & c.(3631-36 p.E1211K & collagen, type XIV, alpha 1 \\
\hline BTC-2 & FBN3 & 84467 & 19 & 8201283 & 8201283 Missense_Mutation & $C$ & $T$ & c. $1334 \mathrm{G}>\mathrm{A}$ & c.(1333-13 p.G445D & fibrillin 3 \\
\hline BTC-2 & FIGN & 55137 & 2 & 164467285 & 164467285 Missense_Mutation & $C$ & A & c. $1057 \mathrm{G}>\mathrm{T}$ & c.(1057-10 p.D353Y & fidgetin \\
\hline BTC-2 & FLNB & 2317 & 3 & 58118580 & 58118580 Missense_Mutation & $A$ & G & c. $4529 A>G$ & c. $(4528-45$ p.H1510R & filamin $B$, beta \\
\hline BTC-2 & INO80D & 54891 & 2 & 206884534 & 206884534 Missense_Mutation & $\mathrm{G}$ & A & c. $1334 C>T$ & c.(1333-13 p.A445V & INO80 complex subunit D \\
\hline BTC-2 & INTS1 & 26173 & 7 & 1544069 & 1544069 Missense_Mutation & $\mathrm{C}$ & G & $c .233 G>C$ & c. $(232-234$ p.C78S & integrator complex subunit 1 \\
\hline BTC-2 & KCNIP1 & 30820 & 5 & 170145809 & 170145809 Missense_Mutation & G & A & c. $109 \mathrm{G}>\mathrm{A}$ & c.(109-111 p.E37K & Kv channel interacting protein 1 \\
\hline BTC-2 & KIAA1549 & 57670 & 7 & 138603864 & 138603864 Missense_Mutation & $\mathrm{G}$ & A & c. $508 \mathrm{C}>\mathrm{T}$ & c.(508-510 p.R170W & KIAA1549 \\
\hline BTC-2 & LGALS9 & 3965 & 17 & 25975970 & 25975970 Missense_Mutation & G & A & c. $934 \mathrm{G}>\mathrm{A}$ & c.(934-936 p.V312M & lectin, galactoside-binding, soluble, 9 \\
\hline BTC-2 & PLEKHG1 & 57480 & 6 & 151152904 & 151152904 Missense_Mutation & G & A & c. $2657 \mathrm{G}>\mathrm{A}$ & c. $(2656-26$ p.R886H & $\begin{array}{l}\text { pleckstrin homology domain containing, family } \mathrm{G} \text { (with RhoGef } \\
\text { domain) member } 1\end{array}$ \\
\hline BTC-2 & PNCK & $139728 X$ & & 152937421 & 152937421 Missense_Mutation & $C$ & G & c. $328 \mathrm{G}>\mathrm{C}$ & c.(328-330 p.E110Q & pregnancy up-regulated nonubiquitous CaM kinase \\
\hline BTC-2 & PPDPF & 79144 & 20 & 62152924 & 62152924 Missense_Mutation & G & A & c. $115 \mathrm{G}>\mathrm{A}$ & c.(115-117 p.A39T & pancreatic progenitor cell differentiation and proliferation factor \\
\hline BTC-2 & PROKR2 & 128674 & 20 & 5282783 & 5282783 Missense_Mutation & $C$ & $\mathrm{~T}$ & c. $1058 \mathrm{G}>\mathrm{A}$ & c. $(1057-10$ p.R353H & prokineticin receptor 2 \\
\hline
\end{tabular}




\begin{tabular}{|c|c|c|c|c|c|c|c|c|c|c|}
\hline BTC-2 & RP11-429E & 0 & 20 & 60294088 & 60294088 Missense_Mutation & G & $A$ & c. $139 C>T$ & c. $(139-141$ p.L47F & \\
\hline BTC-2 & SCN5A & 6331 & 3 & 38645514 & 38645514 Missense_Mutation & $\mathrm{C}$ & $\mathrm{T}$ & c. $1579 G>A$ & c.(1579-15 p.G527R & sodium channel, voltage-gated, type $V$, alpha subunit \\
\hline BTC-2 & SLC35F1 & 222553 & 6 & 118556702 & 118556702 Missense_Mutation & G & A & c. $380 \mathrm{G}>\mathrm{A}$ & c.(379-381 p.R127Q & solute carrier family 35 , member $\mathrm{F} 1$ \\
\hline BTC-2 & SLC9A6 & $10479 x$ & & 135067950 & 135067950 Missense_Mutation & C & $\mathrm{T}$ & c. $289 C>T$ & c. (289-291 p.R97C & $\begin{array}{l}\text { solute carrier family 9, subfamily A (NHE6, cation proton } \\
\text { antiporter } 6 \text { ), member } 6\end{array}$ \\
\hline BTC-2 & SPARC & 6678 & 5 & 151047056 & 151047056 Missense_Mutation & $\mathrm{T}$ & $\mathrm{C}$ & c. $557 \mathrm{~A}>\mathrm{G}$ & c.(556-558 p.N186S & secreted protein, acidic, cysteine-rich (osteonectin) \\
\hline BTC-2 & TLL2 & 7093 & 10 & 98144434 & 98144434 Missense_Mutation & C & G & c. $2104 \mathrm{G}>\mathrm{C}$ & c.(2104-21 p.V702L & tolloid-like 2 \\
\hline BTC-2 & TRIM58 & 25893 & 1 & 248028097 & 248028097 Missense_Mutation & $\mathrm{C}$ & $\mathrm{T}$ & c.607C > T & c.(607-609 p.R203W & tripartite motif containing 58 \\
\hline BTC-2 & TSPAN16 & 26526 & 19 & 11408947 & 11408947 Missense_Mutation & A & G & c. $199 A>G$ & c. $(199-201$ p. $167 \mathrm{~V}$ & tetraspanin 16 \\
\hline BTC-2 & TSSK2 & 23617 & 22 & 19119034 & 19119034 Missense_Mutation & A & C & c. $122 \mathrm{~A}>\mathrm{C}$ & c.(121-123 p.K41T & testis-specific serine kinase 2 \\
\hline BTC-2 & VAV2 & 7410 & 9 & 136635595 & 136635595 Missense_Mutation & G & A & c. $2222 C>T$ & c.(2221-22 p.S741L & vav 2 guanine nucleotide exchange factor \\
\hline BTC-2 & $\mathrm{ZC} 2 \mathrm{HC} 1 \mathrm{~B}$ & 153918 & 6 & 144224199 & 144224199 Missense_Mutation & $\mathrm{C}$ & $\mathrm{T}$ & C. $508 \mathrm{C}>\mathrm{T}$ & c.(508-510 p.P170S & zinc finger, $\mathrm{C} 2 \mathrm{HC}$-type containing $1 \mathrm{~B}$ \\
\hline BTC-2 & ZFP69B & 65243 & 1 & 40928270 & 40928270 Missense_Mutation & $\mathrm{G}$ & $A$ & c. $614 G>A$ & c. $(613-615$ p.G205D & ZFP69 zinc finger protein $B$ \\
\hline BTC-2 & ZNF423 & 23090 & 16 & 49669726 & 49669726 Missense_Mutation & $\mathrm{C}$ & $\mathrm{T}$ & c. $3337 \mathrm{G}>\mathrm{A}$ & c.(3337-33 p.A1113T & zinc finger protein 423 \\
\hline BTC-4 & ARID1A & 8289 & 1 & 27023831 & 27023831 Frame_Shift_Del & G & - & c.937delG & c.(937-939 p.G314fs & AT rich interactive domain $1 \mathrm{~A}$ (SWI-like) \\
\hline BTC-4 & TULP1 & 7287 & 6 & 35480415 & 35480415 Splice_Site & C & G & & c.e $2+1$ & tubby like protein 1 \\
\hline BTC-4 & ACOXL & 55289 & 2 & 111850501 & 111850501 Missense_Mutation & $\mathrm{T}$ & $A$ & c. $1590 \mathrm{~T}>\mathrm{A}$ & c.(1588-15 p.H530Q & acyl-CoA oxidase-like \\
\hline BTC-4 & CACNA1H & 8912 & 16 & 1270626 & 1270626 Missense_Mutation & $\mathrm{C}$ & $A$ & c. $6676 \mathrm{C}>\mathrm{A}$ & c. $(6676-66$ p.L2226M & calcium channel, voltage-dependent, $\mathrm{T}$ type, alpha $1 \mathrm{H}$ subunit \\
\hline BTC-4 & CATSPERB & 79820 & 14 & 92174496 & 92174496 Missense_Mutation & $\mathrm{T}$ & $A$ & c. $455 \mathrm{~A}>\mathrm{T}$ & c.(454-456 p.D152V & catsper channel auxiliary subunit beta \\
\hline BTC-4 & CDKN2A & 1029 & 9 & 21971035 & 21971035 Missense_Mutation & $\mathrm{T}$ & $\mathrm{C}$ & c. $170 A>G$ & c.(169-171 p.D57G & cyclin-dependent kinase inhibitor $2 \mathrm{~A}$ \\
\hline BTC-4 & CES3 & 23491 & 16 & 67006620 & 67006620 Missense_Mutation & $\mathrm{G}$ & A & c. $408 \mathrm{G}>\mathrm{A}$ & c. $(406-408$ p.M136) & carboxylesterase 3 \\
\hline BTC-4 & CLCNKA & 1187 & 1 & 16349137 & 16349137 Missense_Mutation & G & A & c. $23 \mathrm{G}>\mathrm{A}$ & c. $(22-24) c(p . R 8 H$ & chloride channel, voltage-sensitive Ka \\
\hline BTC-4 & COL22A1 & 169044 & 8 & 139890232 & 139890232 Missense_Mutation & C & $\mathrm{T}$ & c. $419 \mathrm{G}>\mathrm{A}$ & c.(418-420 p.R140H & collagen, type XXII, alpha 1 \\
\hline BTC-4 & CRISPLD1 & 83690 & 8 & 75941665 & 75941665 Missense_Mutation & G & $A$ & c. $1364 G>A$ & c.(1363-13 p.R455Q & cysteine-rich secretory protein LCCL domain containing 1 \\
\hline BTC-4 & DAW1 & 164781 & 2 & 228767727 & 228767727 Missense_Mutation & $\mathrm{T}$ & $\mathrm{C}$ & C.550T >C & c.(550-552 p.S184P & dynein assembly factor with WDR repeat domains 1 \\
\hline BTC-4 & DYSF & 8291 & 2 & 71797023 & 71797023 Missense_Mutation & G & $A$ & c. $2938 G>A$ & c.(2938-29. p.G980R & dysferlin \\
\hline BTC-4 & ELN & 2006 & 7 & 73461047 & 73461047 Missense_Mutation & $\mathrm{C}$ & $\mathrm{T}$ & c. $593 C>T$ & c.(592-594 p.P198L & elastin \\
\hline BTC-4 & FAM135B & 51059 & 8 & 139165339 & 139165339 Missense_Mutation & A & G & c.1379T>C & c. $(1378-13$ p.L460P & family with sequence similarity 135 , member $B$ \\
\hline BTC-4 & FAM208B & 54906 & 10 & 5803432 & 5803432 Missense_Mutation & $\mathrm{C}$ & A & c. $7172 \mathrm{C}>\mathrm{A}$ & c.(7171-71 p.T2391K & family with sequence similarity 208 , member $B$ \\
\hline BTC-4 & GPR65 & 8477 & 14 & 88478153 & 88478153 Missense_Mutation & G & $A$ & c. $962 \mathrm{G}>\mathrm{A}$ & c. $(961-963$ p.R321H & G protein-coupled receptor 65 \\
\hline BTC-4 & GUCY1A3 & 2982 & 4 & 156643206 & 156643206 Missense_Mutation & A & $\mathrm{T}$ & c. $1733 \mathrm{~A}>\mathrm{T}$ & c.(1732-17 p.H578L & guanylate cyclase 1 , soluble, alpha 3 \\
\hline BTC-4 & HID1 & 283987 & 17 & 72959068 & 72959068 Missense_Mutation & $\mathrm{T}$ & $A$ & c. $496 \mathrm{~A}>\mathrm{T}$ & c. $(496-498$ p.S166C & HID1 domain containing \\
\hline BTC-4 & HIP1 & 3092 & 7 & 75189111 & 75189111 Missense_Mutation & G & A & c. $1300 C>T$ & c. $(1300-13$ p.R434W & huntingtin interacting protein 1 \\
\hline BTC-4 & IRF3 & 3661 & 19 & 50167939 & 50167939 Missense_Mutation & $\mathrm{T}$ & $\mathrm{C}$ & c. $157 A>G$ & c. $(157-159$ p. $.153 \mathrm{~V}$ & interferon regulatory factor 3 \\
\hline BTC-4 & KCNB1 & 3745 & 20 & 48098906 & 48098906 Missense_Mutation & c & $T$ & c. $112 \mathrm{G}>\mathrm{A}$ & c.(112-114 p.G38R & $\begin{array}{l}\text { potassium voltage-gated channel, Shab-related subfamily, } \\
\text { member } 1\end{array}$ \\
\hline BTC-4 & KCNT1 & 57582 & 9 & 138664603 & 138664603 Missense_Mutation & $\mathrm{C}$ & $\mathrm{T}$ & c. $2051 C>T$ & c.(2050-20 p.T684M & potassium channel, subfamily $T$, member 1 \\
\hline BTC-4 & LLGL1 & 3996 & 17 & 18143990 & 18143990 Missense_Mutation & $\mathrm{C}$ & $\mathrm{T}$ & c. $2305 C>T$ & c.(2305-23 p.R769W & lethal giant larvae homolog 1 (Drosophila) \\
\hline BTC-4 & LPHN1 & 22859 & 19 & 14294398 & 14294398 Missense_Mutation & G & $A$ & c. $17 \mathrm{C}>\mathrm{T}$ & c. $(16-18) g(p . A 6 V$ & latrophilin 1 \\
\hline BTC-4 & MARS & 4141 & 12 & 57906626 & 57906626 Missense_Mutation & A & $\mathrm{T}$ & c. $1846 \mathrm{~A}>\mathrm{T}$ & c. $(1846-18 \cdot p .1616 \mathrm{~F}$ & methionyl-tRNA synthetase \\
\hline BTC-4 & MBTPS1 & 8720 & 16 & 84093019 & 84093019 Missense_Mutation & $\mathrm{G}$ & $A$ & c. $2719 C>T$ & c.(2719-27 p.R907W & membrane-bound transcription factor peptidase, site 1 \\
\hline BTC-4 & PDC & 5132 & 1 & 186413572 & 186413572 Missense_Mutation & $\mathrm{G}$ & $A$ & c. $280 C>T$ & c. $(280-282$ p.R94C & phosducin \\
\hline BTC-4 & PTTG1IP & 754 & 21 & 46285352 & 46285352 Missense_Mutation & C & G & c. $126 \mathrm{G}>\mathrm{C}$ & c.(124-126 p.Q42H & pituitary tumor-transforming 1 interacting protein \\
\hline
\end{tabular}




\begin{tabular}{|c|c|c|c|c|c|}
\hline BTC-4 & RBBP6 & 5930 & 16 & 24581304 & 24581304 Missense_Mutation \\
\hline BTC-4 & RBBP7 & $5931 \times$ & & 16871823 & 16871823 Missense_Mutation \\
\hline BTC-4 & $\mathrm{RDH} 10$ & 157506 & 8 & 74234976 & 74234976 Missense_Mutation \\
\hline BTC-4 & RFX8 & 731220 & 2 & 102018971 & 102018971 Missense_Mutation \\
\hline BTC-4 & SEMA6D & 80031 & 15 & 48058143 & 48058143 Missense_Mutation \\
\hline BTC-4 & SLC6A17 & 388662 & 1 & 110734682 & 110734682 Missense_Mutation \\
\hline BTC-4 & TMEM205 & 374882 & 19 & 11455971 & 11455971 Missense_Mutation \\
\hline BTC-4 & TRIM4 & 89122 & 7 & 99506284 & 99506284 Missense_Mutation \\
\hline BTC-5 & HMCN1 & 83872 & 1 & 186057081 & 186057084 Frame_Shift_Del \\
\hline BTC-5 & UPP2 & 151531 & 2 & 158977957 & 158977958 Frame_Shift_Ins \\
\hline BTC-5 & FNTB & 2342 & 14 & 65511053 & 65511053 Nonsense_Mutation \\
\hline BTC-5 & ACSM2A & 123876 & 16 & 20482871 & 20482871 Nonsense_Mutation \\
\hline BTC-5 & PSD3 & 23362 & 8 & 18430175 & 18430175 Nonsense_Mutation \\
\hline BTC-5 & COL11A1 & 1301 & 1 & 103467527 & 103467527 Splice_Site \\
\hline BTC-5 & AC007405. & 0 & 2 & 171570595 & 171570595 Missense_Mutation \\
\hline BTC-5 & AC009365. & 0 & 7 & 132412557 & 132412557 Missense_Mutation \\
\hline BTC-5 & ADAM21 & 8747 & 14 & 70924614 & 70924614 Missense_Mutation \\
\hline BTC-5 & APOOL & $139322 X$ & & 84329077 & 84329077 Missense_Mutation \\
\hline BTC-5 & ASPM & 259266 & 1 & 197074062 & 197074062 Missense_Mutation \\
\hline BTC-5 & ATP7A & $538 x$ & & 77301051 & 77301051 Missense_Mutation \\
\hline BTC-5 & ATP8B4 & 79895 & 15 & 50288883 & 50288883 Missense_Mutation \\
\hline BTC-5 & CACNA1A & 773 & 19 & 13616812 & 13616812 Missense_Mutation \\
\hline BTC-5 & CCDC175 & 729665 & 14 & 60031860 & 60031860 Missense_Mutation \\
\hline BTC-5 & CCL18 & 6362 & 17 & 34398340 & 34398340 Missense_Mutation \\
\hline BTC-5 & CD163 & 9332 & 12 & 7651580 & 7651580 Missense_Mutation \\
\hline BTC-5 & COL21A1 & 81578 & 6 & 55922544 & 55922544 Missense_Mutation \\
\hline BTC-5 & CSMD3 & 114788 & 8 & 113697935 & 113697935 Missense_Mutation \\
\hline BTC-5 & CXorf28 & $1 \mathrm{E}+08 \mathrm{X}$ & & 3189922 & 3189922 Missense_Mutation \\
\hline BTC-5 & DMXL2 & 23312 & 15 & 51791693 & 51791693 Missense_Mutation \\
\hline BTC-5 & GAS2 & 2620 & 11 & 22747946 & 22747946 Missense_Mutation \\
\hline BTC-5 & GPR98 & 84059 & 5 & 89939702 & 89939702 Missense_Mutation \\
\hline BTC-5 & HOXA4 & 3201 & 7 & 27170310 & 27170310 Missense_Mutation \\
\hline BTC-5 & $\mathrm{HP}$ & 3240 & 16 & 72090131 & 72090131 Missense_Mutation \\
\hline BTC-5 & ICAM5 & 7087 & 19 & 10405259 & 10405259 Missense_Mutation \\
\hline BTC-5 & KIAA1244 & 57221 & 6 & 138576619 & 138576619 Missense_Mutation \\
\hline BTC-5 & KIF21B & 23046 & 1 & 200948693 & 200948693 Missense_Mutation \\
\hline BTC-5 & KRTAP21-1 & 337977 & 21 & 32127554 & 32127554 Missense_Mutation \\
\hline BTC-5 & LCA10 & $0 \times$ & & 153152431 & 153152431 Missense_Mutation \\
\hline
\end{tabular}

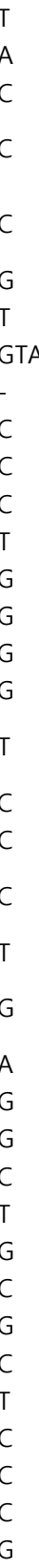

c.3293A $>$ T C.(3292-32 p.E1098V retinoblastoma binding protein 6

c.872A $>$ T c.(871-873 p.D291V retinoblastoma binding protein 7

c.833A $>\mathrm{G}$ c.(832-834 p.K278R retinol dehydrogenase 10 (all-trans)

c.1172G>A c.(1171-11 p.R391Q RFX family member 8, lacking RFX DNA binding domain

c.1505C $>$ T C.(1504-15 p.A502V $\begin{aligned} & \text { sema domain, transmembrane domain (TM), and cytoplasmic } \\ & \text { domain, (semaphorin) } 6 \mathrm{D}\end{aligned}$

c.953C>A C.(952-954 p.A318D $\begin{aligned} & \text { solute carrier family } 6 \text { (neutral amino acid transporter), member } \\ & 17\end{aligned}$

c.221C>T c.(220-222 p.S74L transmembrane protein 205

c.719A $>$ G c. $(718-720$ p.N240S tripartite motif containing 4

c.9381_9384delGT c.(9379-93 p.QY3127f hemicentin 1

c.491_492insT c.(490-495 p.V165fs uridine phosphorylase 2

c.847C $>$ T c. $\quad$ (847-849 p.R283* farnesyltransferase, CAAX box, beta

c.754C $>\mathrm{T} \quad$ c. $(754-756$ p.Q252* acyl-CoA synthetase medium-chain family member $2 \mathrm{~A}$

c.2647G > T c. (2647-26. p.E883* pleckstrin and Sec7 domain containing 3

c.e24-2

collagen, type $\mathrm{XI}$, alpha 1

c. $359 C>\mathrm{G}$

c. $409 \mathrm{G}>\mathrm{A}$

c.398G $>\mathrm{T}$

c. $560 \mathrm{G}>\mathrm{C}$

c.(358-360 p.A120G

c.(409-411 p.V137I

c.(397-399 p.R133L ADAM metallopeptidase domain 21

c.(559-561 p.S187T apolipoprotein O-like

c. $4319 A>G$

c. $4208 \mathrm{C}>\mathrm{A}$

c. $580 \mathrm{G}>\mathrm{C}$

c.(4318-43 p.Q1440R $\begin{aligned} & \text { asp (abnorm } \\ & \text { (Drosophila) }\end{aligned}$

c.(4207-42 p.S1403Y ATPase, Cu++ transporting, alpha polypeptide

c.(580-582 p.G194R ATPase, class I, type 8B, member 4

c. $227 \mathrm{G}>\mathrm{T}$

c. $625 \mathrm{~A}>\mathrm{G}$

c.(226-228 p.R76L calcium channel, voltage-dependent, P/Q type, alpha 1A subunit

c.209G $>A$

c.662T $>C$

c. $2785 \mathrm{C}>\mathrm{A}$

c. $2182 C>G$

c. $49 \mathrm{C}>\mathrm{T}$

c. $3728 \mathrm{~A}>\mathrm{T}$

c.376G $>A$

c. $2636 C>T$

c. $43 \mathrm{C}>\mathrm{A}$

c $77 \mathrm{C}>\mathrm{T}$

c. $2173 \mathrm{~T}>\mathrm{C}$

C. $817 \mathrm{C}>\mathrm{T}$

c. $4130 \mathrm{G}>\mathrm{A}$

c.(625-627 p.1209V coiled-coil domain containing 175

c.(208-210 p.C70Y

chemokine (C-C motif) ligand 18 (pulmonary and activation-

c.(661-663 p.1221T CD163 molecule

c.(2785-27 p.Q929K collagen, type XXI, alpha 1

c.(2182-21 p.P728A CUB and Sushi multiple domains 3

c.(49-51)C(p.P17S chromosome $X$ open reading frame 28

c.(3727-37 p.D1243V Dmx-like 2

c.(376-378 p.D126N growth arrest-specific 2

c.(2635-26 p.T879M G protein-coupled receptor 98

c. (43-45)Cip.P15T homeobox A4

c. (76-78)a(p.T26M haptoglobin

c. (4129-41 p.R1377Q kinesin family member 21B

c. 143G $>T \quad$ c. $(142-144$ p.C48F keratin associated protein 21-1

c.311G $>\mathrm{T} \quad$ c. $(310-312$ p.R104L 


\begin{tabular}{|c|c|c|c|c|c|}
\hline BTC-5 & MAP1B & 4131 & 5 & 71490888 & 71490888 Missense_Mutation \\
\hline BTC-5 & MLK4 & 0 & 1 & 233518335 & 233518335 Missense_Mutation \\
\hline BTC-5 & NBEAL2 & 23218 & 3 & 47041430 & 47041430 Missense_Mutation \\
\hline BTC-5 & NFATC2 & 4773 & 20 & 50140482 & 50140482 Missense_Mutation \\
\hline BTC-5 & NRAS & 4893 & 1 & 115256529 & 115256529 Missense_Mutation \\
\hline BTC-5 & OLFML2B & 25903 & 1 & 161967939 & 161967939 Missense_Mutation \\
\hline BTC-5 & OSBPL5 & 114879 & 11 & 3143583 & 3143583 Missense_Mutation \\
\hline BTC-5 & PCDHB1 & 29930 & 5 & 140432385 & 140432385 Missense_Mutation \\
\hline BTC-5 & PCSK5 & 5125 & 9 & 78936474 & 78936474 Missense_Mutation \\
\hline BTC-5 & PGR & 5241 & 11 & 100998706 & 100998706 Missense_Mutation \\
\hline BTC-5 & PHIP & 55023 & 6 & 79695085 & 79695085 Missense_Mutation \\
\hline BTC-5 & PIK3C2G & 5288 & 12 & 18435656 & 18435656 Missense_Mutation \\
\hline BTC-5 & PRKCD & 5580 & 3 & 53219710 & 53219710 Missense_Mutation \\
\hline BTC-5 & SEC14L2 & 23541 & 22 & 30802345 & 30802345 Missense_Mutation \\
\hline BTC-5 & SEMA6A & 57556 & 5 & 115782872 & 115782872 Missense_Mutation \\
\hline BTC-5 & SKIV2L2 & 23517 & 5 & 54635888 & 54635888 Missense_Mutation \\
\hline BTC-5 & TAF1L & 138474 & 9 & 32631848 & 32631848 Missense_Mutation \\
\hline BTC-5 & TEX15 & 56154 & 8 & 30702546 & 30702546 Missense_Mutation \\
\hline BTC-5 & TLR8 & $51311 x$ & & 12939230 & 12939230 Missense Mutation \\
\hline BTC-5 & TMEM200 & 114801 & 6 & 130762039 & 130762039 Missense_Mutation \\
\hline BTC-5 & TMEM80 & 283232 & 11 & 703112 & 703112 Missense_Mutation \\
\hline BTC-5 & TRPM6 & 140803 & 9 & 77377993 & 77377993 Missense_Mutation \\
\hline BTC-5 & TSPYL5 & 85453 & 8 & 98290039 & 98290039 Missense_Mutation \\
\hline BTC-6 & AMIGO2 & 347902 & 12 & 47471598 & 47471598 Missense_Mutation \\
\hline BTC-6 & BIRC6 & 57448 & 2 & 32712788 & 32712788 Missense_Mutation \\
\hline BTC-6 & BZW1 & 9689 & 2 & 201680210 & 201680210 Missense_Mutation \\
\hline BTC-6 & CD200R1 & 131450 & 3 & 112648303 & 112648303 Missense_Mutation \\
\hline BTC-6 & CRYBG3 & 131544 & 3 & 97593426 & 97593426 Missense_Mutation \\
\hline BTC-6 & DEAF1 & 10522 & 11 & 694992 & 694992 Missense_Mutation \\
\hline BTC-6 & EFCAB5 & 374786 & 17 & 28295933 & 28295933 Missense_Mutation \\
\hline BTC-6 & FSIP2 & 401024 & 2 & 186671383 & 186671383 Missense_Mutation \\
\hline BTC-6 & GAPVD1 & 26130 & 9 & 128064438 & 128064438 Missense_Mutation \\
\hline BTC-6 & IQSEC2 & $23096 \mathrm{X}$ & & 53349985 & 53349985 Missense_Mutation \\
\hline BTC-6 & KDM5A & 5927 & 12 & 427378 & 427378 Missense_Mutation \\
\hline BTC-6 & LPAR3 & 23566 & 1 & 85279695 & 85279695 Missense_Mutation \\
\hline BTC-6 & NLRP1 & 22861 & 17 & 5462767 & 5462767 Missense_Mutation \\
\hline BTC-6 & OR6B3 & 150681 & 2 & 240984973 & 240984973 Missense_Mutation \\
\hline BTC- 6 & OTUD6A & $139562 \times$ & & 69282627 & 69282627 Missense Mutation \\
\hline
\end{tabular}

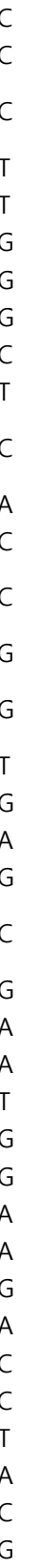

$1706 C>G$

c. $2989 C>T$

c $3841 \mathrm{C}>\mathrm{A}$

c.298G $>A$

c. $182 A>G$

c.1153A>T

c. $295 C>T$

c. $1330 \mathrm{G}>\mathrm{A}$

c.3940G $>A$

c. $1096 \mathrm{G}>\mathrm{A}$

c. $2521 A>C$

c. $641 \mathrm{C}>\mathrm{A}$

c.979A $>\mathrm{T}$

c. $145 \mathrm{C}>\mathrm{G}$

c. $2530 \mathrm{G}>\mathrm{A}$

c.566G $>\mathrm{T}$

c. $3730 \mathrm{C}>\mathrm{T}$

c. $3988 A>C$

c. $2125 G>C$

c. $472 A>G$

c. $469 \mathrm{G}>\mathrm{T}$

c.3594G $>\mathrm{T}$

c. $34 \mathrm{C}>\mathrm{T}$

c. $1188 \mathrm{~T}>\mathrm{G}$

c. $7888 \mathrm{~A}>\mathrm{T}$

c. $211 \mathrm{~T}>\mathrm{G}$

c. $185 C>T$

c. $3388 \mathrm{G}>\mathrm{T}$

c. $56 \mathrm{~T}>\mathrm{C}$

c. $315 \mathrm{~A}>\mathrm{T}$

c. $17350 \mathrm{G}>\mathrm{A}$

c. $362 A>T$

c. $337 \mathrm{G}>\mathrm{A}$

c. $2791 \mathrm{G}>\mathrm{A}$

c. $896 \mathrm{~A}>\mathrm{G}$

c. 1249T >C

C. $517 \mathrm{G}>\mathrm{T}$

c. $253 \mathrm{G}>\mathrm{C}$ c.(1705-17 p.T569R microtubule-associated protein 1B

c.(2989-29 p.P997S

c.(3841-38.p.Q1281K neurobeachin-like 2

c.(298-300 p.A100T nuclear factor of activated T-cells, cytoplasmic, calcineurindependent 2

c.(181-183 p.Q61R neuroblastoma RAS viral (v-ras) oncogene homolog

c.(1153-11 p.S385C olfactomedin-like $2 B$

c.(295-297 p.L99F oxysterol binding protein-like 5

c.(1330-13 p.D444N protocadherin beta 1

c.(3940-39. p.E1314K proprotein convertase subtilisin/kexin type 5

c.(1096-10 p.D366N progesterone receptor

c.(2521-25 p.S841R pleckstrin homology domain interacting protein

c.(640-642 p.P214H phosphatidylinositol-4-phosphate 3-kinase, catalytic subunit type 2 gamma

c.(979-981 p.M327L protein kinase $C$, delta

c.(145-147 p.L49V SEC14-like 2 (S. cerevisiae)

c.(2530-25 p.E844K sema domain, transmembrane domain (TM), and cytoplasmic domain, (semaphorin) $6 \mathrm{~A}$

c.(565-567 p.S189| superkiller viralicidic activity 2-like 2 (S. cerevisiae)

c.(3730-37 p.R1244W TAF1 RNA polymerase II, TATA box binding protein (TBP)associated factor, $210 \mathrm{kDa}$-like

c.(3988-39 p.K1330Q testis expressed 15

c.(2125-21 p.E709Q toll-like receptor 8

c.(472-474 p.1158V transmembrane protein 200A

c.(469-471 p.V157F transmembrane protein 80

c.(3592-35 p.K1198N transient receptor potential cation channel, subfamily M, member

c. $3592-35$ p.K1198N 6

c.(34-36)Cıp.R12C TSPY-like 5

c.(1186-11 p.F396L adhesion molecule with Ig-like domain 2

c.(7888-78 p.I2630F baculoviral IAP repeat containing 6

c.(211-213 p.F71V basic leucine zipper and W2 domains 1

c.(184-186 p.P62L CD200 receptor 1

c.(3388-33 p.G1130W beta-gamma crystallin domain containing 3

c.(55-57)g p.V19A DEAF1 transcription factor

c.(313-315 p.E105D EF-hand calcium binding domain 5

c.(17350-1 p.A5784T fibrous sheath interacting protein 2

c.(361-363 p.N121। GTPase activating protein and VPS9 domains 1

c.337-339 p.D113N IQ motif and Sec7 domain 2

c.(2791-27 p.G931R lysine (K)-specific demethylase 5A

c.(895-897 p.D299G lysophosphatidic acid receptor 3

c.(1249-12 p.W417R NLR family, pyrin domain containing

c.(517-519 p.V173F olfactory receptor, family 6 , subfamily B, member 3

c.(253-255 p.E85Q OTU deubiquitinase 6A 


\begin{tabular}{|c|c|c|c|c|c|}
\hline BTC-6 & PTPRH & 5794 & 19 & 55715264 & 55715264 Missense_Mutation \\
\hline BTC-6 & RAD50 & 10111 & 5 & 131927660 & 131927660 Missense_Mutation \\
\hline BTC-6 & RB1 & 5925 & 13 & 49039417 & 49039417 Missense_Mutation \\
\hline BTC-6 & SAMD9L & 219285 & 7 & 92763376 & 92763376 Missense_Mutation \\
\hline BTC-6 & SERINC5 & 256987 & 5 & 79454696 & 79454696 Missense_Mutation \\
\hline BTC-6 & ST6GALNA & 81849 & 1 & 77515950 & 77515950 Missense_Mutation \\
\hline BTC-6 & TMEM47 & $83604 x$ & & 34657486 & 34657486 Missense_Mutation \\
\hline BTC-6 & TRIM41 & 90933 & 5 & 180651595 & 180651595 Missense_Mutation \\
\hline BTC-6 & WDSUB1 & 151525 & 2 & 160139525 & 160139525 Missense_Mutation \\
\hline BTC-6 & ZC3H13 & 23091 & 13 & 46616352 & 46616352 Missense_Mutation \\
\hline BTC-7 & CCDC18 & 343099 & 1 & 93705341 & 93705341 Nonsense_Mutation \\
\hline BTC-7 & KANSL1 & 284058 & 17 & 44115975 & 44115975 Nonsense_Mutation \\
\hline BTC-7 & PLEKHG2 & 64857 & 19 & 39912773 & 39912773 Nonsense_Mutation \\
\hline BTC-7 & ZNF546 & 339327 & 19 & 40520465 & 40520465 Nonsense_Mutation \\
\hline BTC-7 & SI & 6476 & 3 & 164760855 & 164760855 Nonsense_Mutation \\
\hline BTC-7 & SLITRK2 & $84631 x$ & & 144904042 & 144904042 Nonsense_Mutation \\
\hline BTC-7 & CNTNAP5 & 129684 & 2 & 125367471 & 125367471 Missense_Mutation \\
\hline BTC-7 & DNMT3A & 1788 & 2 & 25467461 & 25467461 Missense_Mutation \\
\hline BTC-7 & DSG4 & 147409 & 18 & 28971077 & 28971077 Missense_Mutation \\
\hline BTC-7 & EPC1 & 80314 & 10 & 32560813 & 32560813 Missense_Mutation \\
\hline BTC-7 & ESM1 & 11082 & 5 & 54277867 & 54277867 Missense_Mutation \\
\hline BTC-7 & F13A1 & 2162 & 6 & 6266956 & 6266956 Missense_Mutation \\
\hline BTC-7 & FCRL3 & 115352 & 1 & 157665154 & 157665154 Missense_Mutation \\
\hline BTC-7 & FUT1 & 2523 & 19 & 49254093 & 49254093 Missense_Mutation \\
\hline BTC-7 & GLI3 & 2737 & 7 & 42005951 & 42005951 Missense_Mutation \\
\hline BTC-7 & HEATR1 & 55127 & 1 & 236721708 & 236721708 Missense_Mutation \\
\hline BTC-7 & KLHL24 & 54800 & 3 & 183368493 & 183368493 Missense_Mutation \\
\hline BTC-7 & KLHL9 & 55958 & 9 & 21333179 & 21333179 Missense_Mutation \\
\hline BTC-7 & КМT2A & 4297 & 11 & 118392628 & 118392628 Missense_Mutation \\
\hline BTC-7 & KRAS & 3845 & 12 & 25398284 & 25398284 Missense_Mutation \\
\hline BTC-7 & LPP & 4026 & 3 & 188326976 & 188326976 Missense_Mutation \\
\hline BTC-7 & LRP1B & 53353 & 2 & 141707920 & 141707920 Missense_Mutation \\
\hline BTC-7 & MSH3 & 4437 & 5 & 79950739 & 79950739 Missense_Mutation \\
\hline BTC-7 & NCOR1 & 9611 & 17 & 16049747 & 16049747 Missense_Mutation \\
\hline BTC-7 & NIPBL & 25836 & 5 & 37064917 & 37064917 Missense_Mutation \\
\hline BTC-7 & NTRK1 & 4914 & 1 & 156851340 & 156851340 Missense_Mutation \\
\hline BTC-7 & PELP1 & 27043 & 17 & 4575146 & 4575146 Missense_Mutation \\
\hline BTC-7 & RELN & 5649 & 7 & 103183183 & 103183183 Missense_Mutation \\
\hline
\end{tabular}




\begin{tabular}{|c|c|c|c|c|c|c|c|c|c|c|}
\hline BTC-7 & SPTBN1 & 6711 & 2 & 54871578 & 54871578 Missense_Mutation & G & $A$ & c. $4124 \mathrm{G}>\mathrm{A}$ & c.(4123-41 p.R1375Q & spectrin, beta, non-erythrocytic 1 \\
\hline BTC-7 & SUSD2 & 56241 & 22 & 24583685 & 24583685 Missense_Mutation & G & $\mathrm{T}$ & $c .2038 G>T$ & c.(2038-20.p.A680S & sushi domain containing 2 \\
\hline BTC-7 & ZNF14 & 7561 & 19 & 19822422 & 19822422 Missense_Mutation & C & A & c.1668G>T & c.(1666-16 p.E556D & zinc finger protein 14 \\
\hline BTC-8 & APC & 324 & 5 & 112175952 & 112175952 Frame_Shift_Del & A & - & c.4661 delA & c. $(4660-46$ p.E1554fs & adenomatous polyposis coli \\
\hline BTC-8 & LMO7 & 4008 & 13 & 76408475 & 76408475 Nonsense_Mutation & C & $\mathrm{T}$ & c. $2479 C>T$ & c. $(2479-24$ p.Q827* & LIM domain 7 \\
\hline BTC-8 & TP53 & 7157 & 17 & 7574003 & 7574003 Nonsense_Mutation & G & A & c. $1024 C>T$ & c.(1024-10 p.R342* & tumor protein p53 \\
\hline BTC-8 & DUXA & 503835 & 19 & 57670637 & 57670637 Nonsense_Mutation & G & $A$ & c. $190 C>T$ & c.(190-192 p.Q64* & double homeobox A \\
\hline BTC-8 & MMS22L & 253714 & 6 & 97676947 & 97676947 Nonsense_Mutation & $C$ & $\mathrm{~T}$ & c. $1862 \mathrm{G}>\mathrm{A}$ & c.(1861-18 p.W621* & MMS22-like, DNA repair protein \\
\hline BTC-8 & KIF15 & 56992 & 3 & 44843467 & 44843467 Splice_Site & G & C & & c.e13+1 & kinesin family member 15 \\
\hline BTC-8 & ABCA9 & 10350 & 17 & 67025361 & 67025361 Missense_Mutation & $\mathrm{T}$ & G & c. $1453 \mathrm{~A}>\mathrm{C}$ & c. $(1453-14$ p.N485H & ATP-binding cassette, sub-family A (ABC1), member 9 \\
\hline BTC-8 & ACSS1 & 84532 & 20 & 25004233 & 25004233 Missense_Mutation & C & $\mathrm{T}$ & c. $676 \mathrm{G}>\mathrm{A}$ & c. $(676-678$ p.G226R & acyl-CoA synthetase short-chain family member 1 \\
\hline BTC-8 & ADAMTS6 & 11174 & 5 & 64595872 & 64595872 Missense_Mutation & G & A & c. $1310 C>T$ & c.(1309-13 p.A437V & ADAM metallopeptidase with thrombospondin type 1 motif, 6 \\
\hline BTC-8 & ADORA1 & 134 & 1 & 203098180 & 203098180 Missense_Mutation & A & G & c. $211 A>G$ & c. $(211-213$ p. $171 \mathrm{~V}$ & adenosine $\mathrm{A} 1$ receptor \\
\hline BTC-8 & $\mathrm{AOC} 3$ & 8639 & 17 & 41006705 & 41006705 Missense_Mutation & C & $\mathrm{T}$ & c. $1841 C>T$ & c.(1840-18 p.P614L & amine oxidase, copper containing 3 \\
\hline BTC-8 & $\mathrm{CPZ}$ & 8532 & 4 & 8603105 & 8603105 Missense_Mutation & G & A & c. $344 G>A$ & c.(343-345 p.R115Q & carboxypeptidase Z \\
\hline BTC-8 & DACT3 & 147906 & 19 & 47151783 & 47151783 Missense_Mutation & G & C & c. $1846 C>G$ & c.(1846-18 p.L616V & dishevelled-binding antagonist of beta-catenin 3 \\
\hline BTC-8 & ENPP2 & 5168 & 8 & 120577135 & 120577135 Missense_Mutation & A & C & c.1097T>G & c. (1096-10 p.V366G & ectonucleotide pyrophosphatase/phosphodiesterase 2 \\
\hline BTC-8 & INO80D & 54891 & 2 & 206870153 & 206870153 Missense_Mutation & C & A & c.2023G>T & c. $(2023-20$ p.A675S & INO80 complex subunit D \\
\hline BTC-8 & IRX3 & 79191 & 16 & 54319378 & 54319378 Missense_Mutation & $\mathrm{T}$ & C & c. $415 A>G$ & c.(415-417 p.T139A & iroquois homeobox 3 \\
\hline BTC-8 & KRAS & 3845 & 12 & 25398281 & 25398281 Missense_Mutation & $\mathrm{C}$ & $\mathrm{T}$ & c.38G $>\mathrm{A}$ & c.(37-39)g(p.G13D & Kirsten rat sarcoma viral oncogene homolog \\
\hline BTC-8 & KRT73 & 319101 & 12 & 53004589 & 53004589 Missense_Mutation & C & $\mathrm{T}$ & c. $1141 \mathrm{G}>\mathrm{A}$ & c.(1141-11.p.A381T & keratin 73 \\
\hline BTC-8 & LAX1 & 54900 & 1 & 203743063 & 203743063 Missense_Mutation & G & A & c. $451 \mathrm{G}>\mathrm{A}$ & c.(451-453 p.A151T & lymphocyte transmembrane adaptor 1 \\
\hline BTC-8 & MATN4 & 8785 & 20 & 43927089 & 43927089 Missense_Mutation & G & A & c. $1147 C>T$ & c.(1147-11.p.R383W & matrilin 4 \\
\hline BTC-8 & NAB1 & 4664 & 2 & 191524551 & 191524551 Missense_Mutation & G & A & c. $649 \mathrm{G}>\mathrm{A}$ & c.(649-651 p.E217K & NGFI-A binding protein 1 (EGR1 binding protein 1) \\
\hline BTC-8 & NPR3 & 4883 & 5 & 32712527 & 32712527 Missense_Mutation & C & A & c. $645 C>A$ & c. (643-645 p.F215L & natriuretic peptide receptor 3 \\
\hline BTC-8 & OTOG & 340990 & 11 & 17655819 & 17655819 Missense_Mutation & A & G & c.7288A>G & c.(7288-72 p.T2430A & otogelin \\
\hline BTC-8 & PCDHGB6 & 56100 & 5 & 140788665 & 140788665 Missense_Mutation & G & A & c. $896 \mathrm{G}>\mathrm{A}$ & c.(895-897 p.G299D & protocadherin gamma subfamily $B, 6$ \\
\hline BTC-8 & POLA1 & $5422 x$ & & 24906154 & 24906154 Missense_Mutation & G & A & c. $4061 \mathrm{G}>\mathrm{A}$ & c. $(4060-40$ p.R1354H & polymerase (DNA directed), alpha 1, catalytic subunit \\
\hline BTC-8 & RALGAPA1 & 253959 & 14 & 36096402 & 36096402 Missense_Mutation & $\mathrm{T}$ & A & c. $5272 A>T$ & c.(5272-52 p.M1758L & Ral GTPase activating protein, alpha subunit 1 (catalytic) \\
\hline BTC-8 & RIPK2 & 8767 & 8 & 90792331 & 90792331 Missense_Mutation & G & $\mathrm{C}$ & c. $882 \mathrm{G}>\mathrm{C}$ & c. (880-882 p.L294F & receptor-interacting serine-threonine kinase 2 \\
\hline BTC-8 & ROBO1 & 6091 & 3 & 78710287 & 78710287 Missense_Mutation & A & G & c. $2213 T>C$ & c.(2212-22 p.1738T & roundabout, axon guidance receptor, homolog 1 (Drosophila) \\
\hline BTC-8 & $\mathrm{ROBO} 2$ & 6092 & 3 & 77657029 & 77657029 Missense_Mutation & C & A & c.3217C>A & c.(3217-32 p.P1073T & roundabout, axon guidance receptor, homolog 2 (Drosophila) \\
\hline BTC-8 & SHISA8 & 440829 & 22 & 42310285 & 42310285 Missense_Mutation & C & A & c. $286 G>T$ & c. (286-288 p.A96S & shisa family member 8 \\
\hline BTC-8 & SMARCA2 & 6595 & 9 & 2104152 & 2104152 Missense_Mutation & $\mathrm{T}$ & c & c.3275T>C & c.(3274-32 p.L1092P & $\begin{array}{l}\text { SWI/SNF related, matrix associated, actin dependent regulator of } \\
\text { chromatin, subfamily a, member } 2\end{array}$ \\
\hline BTC-8 & SSTR1 & 6751 & 14 & 38678956 & 38678956 Missense_Mutation & G & A & c. $362 \mathrm{G}>\mathrm{A}$ & c. $(361-363$ p.R121H & somatostatin receptor 1 \\
\hline BTC-8 & SYN3 & 8224 & 22 & 33265027 & 33265027 Missense_Mutation & C & $\mathrm{T}$ & c. $547 \mathrm{G}>\mathrm{A}$ & c.(547-549 p.G183S & synapsin III \\
\hline BTC-8 & TROVE2 & 6738 & 1 & 193046115 & 193046115 Missense_Mutation & $\mathrm{C}$ & G & c.1021C>G & c.(1021-10 p.L341V & TROVE domain family, member 2 \\
\hline BTC-8 & USH2A & 7399 & 1 & 215844423 & 215844423 Missense_Mutation & C & $\mathrm{T}$ & c. $14024 \mathrm{G}>\mathrm{A}$ & c.(14023-1. p.R4675K & Usher syndrome $2 \mathrm{~A}$ (autosomal recessive, mild) \\
\hline BTC-8 & USH2A & 7399 & 1 & 216062042 & 216062042 Missense_Mutation & G & $\mathrm{C}$ & c. $7949 C>G$ & c.(7948-79 p.P2650R & Usher syndrome 2A (autosomal recessive, mild) \\
\hline BTC-8 & XCR1 & 2829 & 3 & 46062623 & 46062623 Missense_Mutation & G & A & c. $817 C>T$ & c. $(817-819$ p.R273C & chemokine ( $\mathrm{C}$ motif) receptor 1 \\
\hline BTC-8 & ZSCAN20 & 7579 & 1 & 33960845 & 33960845 Missense_Mutation & $\mathrm{C}$ & A & c. $2901 C>A$ & c.(2899-29 p.F967L & zinc finger and SCAN domain containing 20 \\
\hline
\end{tabular}




\section{Supplementary Table 1. Coverage Information}

\begin{tabular}{|c|c|c|c|c|c|c|c|c|c|c|}
\hline Sample & $\mathrm{X} 1$ & $\times 5$ & X10 & $\mathrm{X} 15$ & X20 & $\mathrm{X} 25$ & X30 & $\times 50$ & $\begin{array}{l}\text { Total bases } \\
\text { aligned(bases) }\end{array}$ & $\begin{array}{l}\text { Mean coverage } \\
\text { depth }\end{array}$ \\
\hline BTC_1N & $99.96 \%$ & $99.77 \%$ & $99.22 \%$ & $98.14 \%$ & $96.33 \%$ & $93.69 \%$ & $90.19 \%$ & $69.94 \%$ & $6,034,974,927$ & 80.99 \\
\hline BTC_1T & $99.97 \%$ & $99.90 \%$ & $99.71 \%$ & $99.38 \%$ & $98.84 \%$ & $98.03 \%$ & $96.92 \%$ & $89.02 \%$ & $9,863,779,665$ & 132.38 \\
\hline BTC_2N & $99.86 \%$ & $99.66 \%$ & $99.15 \%$ & $98.17 \%$ & $96.48 \%$ & $93.95 \%$ & $90.45 \%$ & $69.62 \%$ & $5,978,637,815$ & 80.24 \\
\hline BTC_2T & $99.88 \%$ & $99.79 \%$ & $99.63 \%$ & $99.38 \%$ & $99.00 \%$ & $98.46 \%$ & $97.71 \%$ & $92.10 \%$ & $11,086,728,430$ & 148.79 \\
\hline BTC_4N & $99.97 \%$ & $99.80 \%$ & $99.30 \%$ & $98.32 \%$ & $96.67 \%$ & $94.26 \%$ & $91.07 \%$ & $72.14 \%$ & $6,345,622,329$ & 85.16 \\
\hline BTC_4T & $99.98 \%$ & $99.90 \%$ & $99.72 \%$ & $99.43 \%$ & $98.98 \%$ & $98.33 \%$ & $97.44 \%$ & $91.14 \%$ & $10,760,737,151$ & 144.41 \\
\hline BTC_5N & $99.86 \%$ & $99.62 \%$ & $98.95 \%$ & $97.66 \%$ & $95.53 \%$ & $92.44 \%$ & $88.40 \%$ & $66.57 \%$ & $5,823,253,443$ & 78.15 \\
\hline BTC_5T & $99.87 \%$ & $99.78 \%$ & $99.57 \%$ & $99.21 \%$ & $98.65 \%$ & $97.88 \%$ & $96.83 \%$ & $89.50 \%$ & $10,599,020,827$ & 142.24 \\
\hline BTC_6N & $99.87 \%$ & $99.67 \%$ & $99.16 \%$ & $98.14 \%$ & $96.43 \%$ & $93.80 \%$ & $90.22 \%$ & $69.15 \%$ & $5,968,127,744$ & 80.09 \\
\hline BTC_6T & $99.88 \%$ & $99.79 \%$ & $99.63 \%$ & $99.37 \%$ & $98.99 \%$ & $98.43 \%$ & $97.66 \%$ & $91.94 \%$ & $10,923,830,027$ & 146.60 \\
\hline BTC_7N & $99.97 \%$ & $99.78 \%$ & $99.25 \%$ & $98.21 \%$ & $96.48 \%$ & $93.96 \%$ & $90.63 \%$ & $71.11 \%$ & $6,186,543,991$ & 83.03 \\
\hline BTC_7T & $99.98 \%$ & $99.88 \%$ & $99.66 \%$ & $99.27 \%$ & $98.66 \%$ & $97.75 \%$ & $96.48 \%$ & $87.79 \%$ & $9,956,458,889$ & 133.62 \\
\hline BTC_8N & $99.97 \%$ & $99.76 \%$ & $99.17 \%$ & $97.97 \%$ & $95.96 \%$ & $93.09 \%$ & $89.35 \%$ & $68.81 \%$ & $5,998,051,912$ & 80.50 \\
\hline BTC_8T & $99.98 \%$ & $99.89 \%$ & $99.68 \%$ & $99.34 \%$ & $98.82 \%$ & $98.06 \%$ & $97.01 \%$ & $89.49 \%$ & $10,131,605,777$ & 135.97 \\
\hline
\end{tabular}




\section{Supplementary Table 2. Detailed information on total somatic mutations}

\begin{tabular}{|c|c|c|c|c|c|c|c|c|c|c|c|}
\hline Patient ID & Hugo_Sym Er & ntrez_Gen Ch & noso & art_position & End_position & Variant_Classification & Reference_ & -Tumor_Sec & q cDNA_Change & Codon_ChiProtein_Cr & hescription \\
\hline BTC-1 & APC & 324 & 5 & 112174759 & 112174760 & Frame_Shift_Del & AG & - & c.3468_3469delA & C.c.(3466-34 p.EE1156fs & s adenomatous polyposis coli \\
\hline BTC-1 & KRTAP19- $\epsilon$ & 337973 & 21 & 31914024 & 31914024 & Nonsense_Mutation & G & $\mathrm{T}$ & c. $129 C>A$ & c.(127-129 p.C43* & keratin associated protein $19-6$ \\
\hline BTC-1 & PABPC4L & 132430 & 4 & 135121319 & 135121319 & Nonsense_Mutation & G & A & $\mathrm{c} .856 \mathrm{C}>\mathrm{T}$ & c. $(856-858$ p.R286* & poly(A) binding protein, cytoplasmic 4-like \\
\hline BTC-1 & APC & 324 & 5 & 112175951 & 112175951 & Nonsense_Mutation & G & $\mathrm{T}$ & $c .4660 G>T$ & c. $(4660-46$ p.E1554* & adenomatous polyposis coli \\
\hline BTC-1 & C6orf222 & 389384 & 6 & 36290159 & 36290159 & Nonsense_Mutation & G & c & c. $1532 C>G$ & c.(1531-15 p.S511* & chromosome 6 open reading frame 222 \\
\hline BTC-1 & $\mathrm{ABCA} 1$ & 19 & 9 & 107553269 & 107553269 & Nonsense_Mutation & G & C & c. $5861 C>G$ & c.(5860-58 p.S1954* & ATP-binding cassette, sub-family $A(A B C 1)$, member 1 \\
\hline BTC-1 & AMER1 & $139285 X$ & & 63412530 & 63412530 & Nonsense_Mutation & G & A & c. $637 \mathrm{C}>\mathrm{T}$ & c.(637-639 p.Q213* & APC membrane recruitment protein 1 \\
\hline BTC-1 & MFAP2 & 4237 & 1 & 17302995 & 17302995 & Splice_Site & $\mathrm{C}$ & A & & c.e5+1 & microfibrillar-associated protein 2 \\
\hline BTC-1 & HIF3A & 64344 & 19 & 46825222 & 46825222 & Splice_Site & C & $\mathrm{T}$ & c. $1334 C>T$ & c.(1333-13 p.S445L & hypoxia inducible factor 3 , alpha subunit \\
\hline BTC-1 & MAP3K13 & 9175 & 3 & 185155234 & 185155234 & Splice_Site & G & C & & c.e3-1 & mitogen-activated protein kinase kinase kinase 13 \\
\hline BTC-1 & DAB2IP & 153090 & 9 & 124329507 & 124329507 & Splice_Site & G & A & c. $40 \mathrm{G}>\mathrm{A}$ & c.(40-42)Gip.E14K & DAB2 interacting protein \\
\hline BTC-1 & $\mathrm{ABCC} 2$ & 1244 & 10 & 101591752 & 101591752 & Missense_Mutation & C & T & c. $3122 C>T$ & c.(3121-31 p.A1041V & ATP-binding cassette, sub-family C (CFTR/MRP), member 2 \\
\hline BTC-1 & ACE & 1636 & 17 & 61570945 & 61570945 & Missense_Mutation & G & A & c. $1339 \mathrm{G}>\mathrm{A}$ & c.(1339-13.p.V447M & angiotensin I converting enzyme \\
\hline BTC-1 & ACTN2 & 88 & 1 & 236899007 & 236899007 & Missense_Mutation & C & T & c. $770 \mathrm{C}>\mathrm{T}$ & c.(769-771 p.A257V & actinin, alpha 2 \\
\hline BTC-1 & ACTN4 & 81 & 19 & 39207832 & 39207832 & Missense_Mutation & G & A & c. $1019 \mathrm{G}>\mathrm{A}$ & c.(1018-10 p.R340H & actinin, alpha 4 \\
\hline BTC-1 & ACVR1B & 91 & 12 & 52379016 & 52379016 & Missense_Mutation & C & A & c. $864 C>A$ & c.(862-864 p.N288K & activin $A$ receptor, type IB \\
\hline BTC-1 & ADCY5 & 111 & 3 & 123166420 & 123166420 & Missense_Mutation & $\mathrm{C}$ & T & c. $973 \mathrm{G}>\mathrm{A}$ & c.(973-975 p.A325T & adenylate cyclase 5 \\
\hline BTC-1 & ADCY8 & 114 & 8 & 131949376 & 131949376 & Missense_Mutation & C & $\mathrm{T}$ & c. $1424 G>A$ & c.(1423-14 p.R475H & adenylate cyclase 8 (brain) \\
\hline BTC-1 & ANO3 & 63982 & 11 & 26463533 & 26463533 & Missense_Mutation & $\mathrm{T}$ & $\mathrm{C}$ & C. $115 T>C$ & c. $(115-117$ p.C39R & anoctamin 3 \\
\hline BTC-1 & APC2 & 10297 & 19 & 1457029 & 1457029 & Missense_Mutation & G & A & c. $994 \mathrm{G}>\mathrm{A}$ & c.(994-996 p.A332T & adenomatosis polyposis coli 2 \\
\hline BTC-1 & AQPEP & 0 & 5 & 115298621 & 115298621 & Missense_Mutation & G & A & c. $307 \mathrm{G}>\mathrm{A}$ & c.(307-309 p.V103M & \\
\hline BTC-1 & AUTS2 & 26053 & 7 & 69364311 & 69364311 & Missense_Mutation & $\mathrm{C}$ & T & c. $349 C>T$ & c.(349-351 p.R117C & autism susceptibility candidate 2 \\
\hline BTC-1 & C11orf63 & 79864 & 11 & 122775018 & 122775018 & Missense_Mutation & C & $\mathrm{T}$ & c.730C $>\mathrm{T}$ & c.(730-732 p.R244C & chromosome 11 open reading frame 63 \\
\hline BTC-1 & C5orf42 & 65250 & 5 & 37153858 & 37153858 & Missense_Mutation & $\mathrm{T}$ & A & c. $8195 \mathrm{~A}>\mathrm{T}$ & c.(8194-81 p.D2732V & chromosome 5 open reading frame 42 \\
\hline BTC-1 & $\mathrm{CDH} 2$ & 1000 & 18 & 25572711 & 25572711 & Missense_Mutation & C & $\mathrm{T}$ & c. $1159 \mathrm{G}>\mathrm{A}$ & c.(1159-11 p.A387T & cadherin 2 , type $1, \mathrm{~N}$-cadherin (neuronal) \\
\hline BTC-1 & CHST9 & 83539 & 18 & 24628462 & 24628462 & Missense_Mutation & $\mathrm{C}$ & $\mathrm{T}$ & c. $127 \mathrm{G}>\mathrm{A}$ & c. $(127-129$ p.V43M & carbohydrate ( $\mathrm{N}$-acetylgalactosamine 4-0) sulfotransferase 9 \\
\hline BTC-1 & CYP11B1 & 1584 & 8 & 143956558 & 143956558 & Missense_Mutation & C & T & c. $1426 \mathrm{G}>\mathrm{A}$ & c.(1426-14 p.V476M & cytochrome P450, family 11 , subfamily B, polypeptide 1 \\
\hline BTC-1 & DNAH10 & 196385 & 12 & 124341718 & 124341718 & Missense_Mutation & G & A & c. $6200 \mathrm{G}>\mathrm{A}$ & c. $(6199-62$ p.R2067H & dynein, axonemal, heavy chain 10 \\
\hline BTC-1 & DSCAM & 1826 & 21 & 41559851 & 41559851 & Missense_Mutation & $\mathrm{C}$ & $\mathrm{T}$ & c. $2617 G>A$ & c.(2617-26 p.E873K & Down syndrome cell adhesion molecule \\
\hline BTC-1 & ELF3 & 1999 & 1 & 201983140 & 201983140 & Missense_Mutation & G & A & c. $989 \mathrm{G}>\mathrm{A}$ & c.(988-990 p.S330N & $\begin{array}{l}\text { E74-like factor } 3 \text { (ets domain transcription factor, epithelial- } \\
\text { specific ) }\end{array}$ \\
\hline BTC-1 & FGD3 & 89846 & 9 & 95738639 & 95738639 & Missense_Mutation & C & T & c. $101 C>T$ & c. $(100-102$ p.A34V & FYVE, RhoGEF and PH domain containing 3 \\
\hline BTC-1 & FUBP3 & 8939 & 9 & 133493210 & 133493210 & Missense_Mutation & G & A & c. $594 \mathrm{G}>\mathrm{A}$ & c.(592-594 p.M198I & far upstream element (FUSE) binding protein 3 \\
\hline BTC-1 & HAND1 & 9421 & 5 & 153855452 & 153855452 & Missense_Mutation & G & T & c. $562 C>A$ & c. (562-564 p.P188T & heart and neural crest derivatives expressed 1 \\
\hline BTC-1 & KCNS2 & 3788 & 8 & 99440299 & 99440299 & Missense_Mutation & G & A & c. $92 \mathrm{G}>\mathrm{A}$ & c. $(91-93) c(p . R 31 \mathrm{H}$ & $\begin{array}{l}\text { potassium voltage-gated channel, delayed-rectifier, subfamily } S \text {, } \\
\text { member } 2\end{array}$ \\
\hline BTC-1 & KIAA1429 & 25962 & 8 & 95503865 & 95503865 & Missense_Mutation & $\mathrm{C}$ & G & c. $5081 G>C$ & c.(5080-50 p.R1694T & KIAA1429 \\
\hline BTC-1 & KRTAP19-1 & 337882 & 21 & 31852374 & 31852374 & Missense_Mutation & $\mathrm{C}$ & T & c. $263 \mathrm{G}>\mathrm{A}$ & c. $(262-264$ p.G88D & keratin associated protein $19-1$ \\
\hline BTC-1 & LRP1B & 53353 & 2 & 141004693 & 141004693 & Missense_Mutation & G & T & c. $13286 C>A$ & c.(13285-1 p.P4429Q & low density lipoprotein receptor-related protein 1B \\
\hline BTC-1 & LRRK2 & 120892 & 12 & 40689336 & 40689336 & Missense_Mutation & G & $\mathrm{T}$ & c. $2986 G>T$ & c.(2986-29 p.D996Y & leucine-rich repeat kinase 2 \\
\hline BTC-1 & MAP2K1 & 5604 & 15 & 66727455 & 66727455 & Missense_Mutation & G & C & c. $171 \mathrm{G}>\mathrm{C}$ & c. $(169-171$ p.K57N & mitogen-activated protein kinase kinase 1 \\
\hline
\end{tabular}




\begin{tabular}{|c|c|c|c|c|c|c|c|c|c|c|}
\hline BTC-1 & MBTD1 & 54799 & 17 & 49296318 & 49296318 Missense_Mutation & $A$ & $C$ & c. $376 \mathrm{~T}>\mathrm{G}$ & c.(376-378 p.L126V & mbt domain containing 1 \\
\hline BTC-1 & MUC19 & 283463 & 12 & 40820354 & 40820354 Missense_Mutation & G & A & c. $332 \mathrm{G}>\mathrm{A}$ & c.(331-333 p.R111H & mucin 19 , oligomeric \\
\hline BTC-1 & MYLK2 & 85366 & 20 & 30419645 & 30419645 Missense_Mutation & $\mathrm{G}$ & A & c. $1564 G>A$ & c.(1564-15 p.V522I & myosin light chain kinase 2 \\
\hline BTC-1 & PCDH17 & 27253 & 13 & 58207860 & 58207860 Missense_Mutation & $\mathrm{G}$ & A & c. $1180 G>A$ & c.(1180-11 p.G394R & protocadherin 17 \\
\hline BTC-1 & PLIN4 & 729359 & 19 & 4510530 & 4510530 Missense_Mutation & $\mathrm{G}$ & A & c. $3400 C>T$ & c.(3400-34 p.R1134C & perilipin 4 \\
\hline BTC-1 & PRRC2B & 84726 & 9 & 134322013 & 134322013 Missense_Mutation & $A$ & G & $c .839 A>G$ & c.(838-840 p.D280G & proline-rich coiled-coil 2B \\
\hline BTC-1 & RBM15 & 64783 & 1 & 110883546 & 110883546 Missense_Mutation & $\mathrm{T}$ & G & c.1519T>G & c.(1519-15 p.W507G & RNA binding motif protein 15 \\
\hline BTC-1 & RP1-139D & 0 & 6 & 42123292 & 42123292 Missense_Mutation & $\mathrm{G}$ & T & c. $14 \mathrm{G}>\mathrm{T}$ & c. $(13-15) a(p . S 5 \mid$ & \\
\hline BTC-1 & SDK1 & 221935 & 7 & 4273010 & 4273010 Missense_Mutation & $\mathrm{C}$ & $\mathrm{T}$ & c. $5891 C>T$ & c.(5890-58 p.A1964V & sidekick cell adhesion molecule 1 \\
\hline BTC-1 & SPRY2 & 10253 & 13 & 80911584 & 80911584 Missense_Mutation & $\mathrm{G}$ & C & c. $257 C>G$ & c.(256-258 p.P86R & sprouty homolog 2 (Drosophila) \\
\hline BTC-1 & ST6GALNA & 81849 & 1 & 77509964 & 77509964 Missense_Mutation & $C$ & A & c. $337 C>A$ & c.(337-339 p.Q113K & $\begin{array}{l}\text { ST6 (alpha- } \mathrm{N} \text {-acetyl-neuraminyl-2,3-beta-galactosyl-1,3)- } \mathrm{N} \text { - } \\
\text { acetylgalactosaminide alpha-2,6-sialyltransferase } 5\end{array}$ \\
\hline BTC-1 & SYT3 & 84258 & 19 & 51135883 & 51135883 Missense_Mutation & $\mathrm{C}$ & $\mathrm{T}$ & c. $334 \mathrm{G}>\mathrm{A}$ & c.(334-336 p.G112R & synaptotagmin III \\
\hline BTC-1 & TADA2A & 6871 & 17 & 35837062 & 35837062 Missense_Mutation & G & C & c. $1307 \mathrm{G}>\mathrm{C}$ & c.(1306-13 p.R436T & transcriptional adaptor $2 \mathrm{~A}$ \\
\hline BTC-1 & TGM2 & 7052 & 20 & 36793594 & 36793594 Missense_Mutation & $\mathrm{C}$ & $\mathrm{T}$ & $c .7 \mathrm{G}>\mathrm{A}$ & c. (7-9)Gag p.E3K & transglutaminase 2 \\
\hline BTC-1 & TMEM184I & 25829 & 22 & 38643871 & 38643871 Missense_Mutation & $\mathrm{T}$ & C & c. $97 \mathrm{~A}>\mathrm{G}$ & c.(97-99)Аср.ТЗ3А & transmembrane protein $184 \mathrm{~B}$ \\
\hline BTC-1 & TRPC1 & 7220 & 3 & 142499703 & 142499703 Missense_Mutation & A & $\mathrm{T}$ & c.792A $>\mathrm{T}$ & c.(790-792 p.Q264H & transient receptor potential cation channel, subfamily $C$, member \\
\hline BTC-1 & TSHZ3 & 57616 & 19 & 31770589 & 31770589 Missense_Mutation & G & C & c. $110 C>G$ & c.(109-111 p.A37G & teashirt zinc finger homeobox 3 \\
\hline BTC-1 & TSPAN14 & 81619 & 10 & 82271948 & 82271948 Missense_Mutation & $\mathrm{T}$ & G & c.499T > G & c.(499-501 p.Y167D & tetraspanin 14 \\
\hline BTC-1 & ZFR & 51663 & 5 & 32395347 & 32395347 Missense_Mutation & C & $\mathrm{T}$ & c. $1897 \mathrm{G}>\mathrm{A}$ & c.(1897-18 p.E633K & zinc finger RNA binding protein \\
\hline BTC-1 & ZFYVE26 & 23503 & 14 & 68264958 & 68264958 Missense_Mutation & $C$ & A & c. $2021 \mathrm{G}>\mathrm{T}$ & c.(2020-20 p.G674V & zinc finger, FYVE domain containing 26 \\
\hline BTC-2 & EPHA2 & 1969 & 1 & 16464447 & 16464447 Frame_Shift_Del & G & - & c.1213delC & c.(1213-12 p.H405fs & EPH receptor A2 \\
\hline BTC-2 & LGI4 & 163175 & 19 & 35617610 & 35617610 Nonsense_Mutation & $\mathrm{C}$ & $\mathrm{T}$ & $c .863 \mathrm{G}>\mathrm{A}$ & c.(862-864 p.W288* & leucine-rich repeat LGI family, member 4 \\
\hline BTC-2 & EPHA2 & 1969 & 1 & 16456720 & 16456720 Splice_Site & $C$ & $\mathrm{~T}$ & & c.e15+1 & $\mathrm{EPH}$ receptor $\mathrm{A} 2$ \\
\hline BTC-2 & C17orf97 & 400566 & 17 & 260315 & 260315 Missense_Mutation & G & $\mathrm{T}$ & c. $182 \mathrm{G}>\mathrm{T}$ & c.(181-183 p.G61V & chromosome 17 open reading frame 97 \\
\hline BTC-2 & CAMK2N1 & 55450 & 1 & 20810156 & 20810156 Missense_Mutation & G & A & c. $223 C>T$ & c.(223-225 p.P75S & calcium/calmodulin-dependent protein kinase II inhibitor 1 \\
\hline BTC-2 & CNBD1 & 168975 & 8 & 88249206 & 88249206 Missense_Mutation & G & A & c. $637 \mathrm{G}>\mathrm{A}$ & c. (637-639 p.V213M & cyclic nucleotide binding domain containing 1 \\
\hline BTC-2 & COL14A1 & 7373 & 8 & 121292323 & 121292323 Missense_Mutation & $\mathrm{G}$ & A & c. $3631 \mathrm{G}>\mathrm{A}$ & c.(3631-36 p.E1211K & collagen, type XIV, alpha 1 \\
\hline BTC-2 & FBN3 & 84467 & 19 & 8201283 & 8201283 Missense_Mutation & $C$ & $T$ & c. $1334 \mathrm{G}>\mathrm{A}$ & c.(1333-13 p.G445D & fibrillin 3 \\
\hline BTC-2 & FIGN & 55137 & 2 & 164467285 & 164467285 Missense_Mutation & $C$ & A & c. $1057 \mathrm{G}>\mathrm{T}$ & c.(1057-10 p.D353Y & fidgetin \\
\hline BTC-2 & FLNB & 2317 & 3 & 58118580 & 58118580 Missense_Mutation & $A$ & G & c. $4529 A>G$ & c. $(4528-45$ p.H1510R & filamin $B$, beta \\
\hline BTC-2 & INO80D & 54891 & 2 & 206884534 & 206884534 Missense_Mutation & $\mathrm{G}$ & A & c. $1334 C>T$ & c.(1333-13 p.A445V & INO80 complex subunit D \\
\hline BTC-2 & INTS1 & 26173 & 7 & 1544069 & 1544069 Missense_Mutation & $\mathrm{C}$ & G & $c .233 G>C$ & c. $(232-234$ p.C78S & integrator complex subunit 1 \\
\hline BTC-2 & KCNIP1 & 30820 & 5 & 170145809 & 170145809 Missense_Mutation & G & A & c. $109 \mathrm{G}>\mathrm{A}$ & c.(109-111 p.E37K & Kv channel interacting protein 1 \\
\hline BTC-2 & KIAA1549 & 57670 & 7 & 138603864 & 138603864 Missense_Mutation & $\mathrm{G}$ & A & c. $508 \mathrm{C}>\mathrm{T}$ & c.(508-510 p.R170W & KIAA1549 \\
\hline BTC-2 & LGALS9 & 3965 & 17 & 25975970 & 25975970 Missense_Mutation & G & A & c. $934 \mathrm{G}>\mathrm{A}$ & c.(934-936 p.V312M & lectin, galactoside-binding, soluble, 9 \\
\hline BTC-2 & PLEKHG1 & 57480 & 6 & 151152904 & 151152904 Missense_Mutation & G & A & c. $2657 \mathrm{G}>\mathrm{A}$ & c. $(2656-26$ p.R886H & $\begin{array}{l}\text { pleckstrin homology domain containing, family } \mathrm{G} \text { (with RhoGef } \\
\text { domain) member } 1\end{array}$ \\
\hline BTC-2 & PNCK & $139728 X$ & & 152937421 & 152937421 Missense_Mutation & $C$ & G & c. $328 \mathrm{G}>\mathrm{C}$ & c.(328-330 p.E110Q & pregnancy up-regulated nonubiquitous CaM kinase \\
\hline BTC-2 & PPDPF & 79144 & 20 & 62152924 & 62152924 Missense_Mutation & G & A & c. $115 \mathrm{G}>\mathrm{A}$ & c.(115-117 p.A39T & pancreatic progenitor cell differentiation and proliferation factor \\
\hline BTC-2 & PROKR2 & 128674 & 20 & 5282783 & 5282783 Missense_Mutation & $C$ & $\mathrm{~T}$ & c. $1058 \mathrm{G}>\mathrm{A}$ & c. $(1057-10$ p.R353H & prokineticin receptor 2 \\
\hline
\end{tabular}




\begin{tabular}{|c|c|c|c|c|c|c|c|c|c|c|}
\hline BTC-2 & RP11-429E & 0 & 20 & 60294088 & 60294088 Missense_Mutation & G & $A$ & c. $139 C>T$ & c. $(139-141$ p.L47F & \\
\hline BTC-2 & SCN5A & 6331 & 3 & 38645514 & 38645514 Missense_Mutation & $\mathrm{C}$ & $\mathrm{T}$ & c. $1579 G>A$ & c.(1579-15 p.G527R & sodium channel, voltage-gated, type $V$, alpha subunit \\
\hline BTC-2 & SLC35F1 & 222553 & 6 & 118556702 & 118556702 Missense_Mutation & G & A & c. $380 \mathrm{G}>\mathrm{A}$ & c.(379-381 p.R127Q & solute carrier family 35 , member $\mathrm{F} 1$ \\
\hline BTC-2 & SLC9A6 & $10479 x$ & & 135067950 & 135067950 Missense_Mutation & C & $\mathrm{T}$ & c. $289 C>T$ & c. (289-291 p.R97C & $\begin{array}{l}\text { solute carrier family 9, subfamily A (NHE6, cation proton } \\
\text { antiporter } 6 \text { ), member } 6\end{array}$ \\
\hline BTC-2 & SPARC & 6678 & 5 & 151047056 & 151047056 Missense_Mutation & $\mathrm{T}$ & $\mathrm{C}$ & c. $557 \mathrm{~A}>\mathrm{G}$ & c.(556-558 p.N186S & secreted protein, acidic, cysteine-rich (osteonectin) \\
\hline BTC-2 & TLL2 & 7093 & 10 & 98144434 & 98144434 Missense_Mutation & C & G & c. $2104 \mathrm{G}>\mathrm{C}$ & c.(2104-21 p.V702L & tolloid-like 2 \\
\hline BTC-2 & TRIM58 & 25893 & 1 & 248028097 & 248028097 Missense_Mutation & $\mathrm{C}$ & $\mathrm{T}$ & c.607C > T & c.(607-609 p.R203W & tripartite motif containing 58 \\
\hline BTC-2 & TSPAN16 & 26526 & 19 & 11408947 & 11408947 Missense_Mutation & A & G & c. $199 A>G$ & c. $(199-201$ p. $167 \mathrm{~V}$ & tetraspanin 16 \\
\hline BTC-2 & TSSK2 & 23617 & 22 & 19119034 & 19119034 Missense_Mutation & A & C & c. $122 \mathrm{~A}>\mathrm{C}$ & c.(121-123 p.K41T & testis-specific serine kinase 2 \\
\hline BTC-2 & VAV2 & 7410 & 9 & 136635595 & 136635595 Missense_Mutation & G & A & c. $2222 C>T$ & c.(2221-22 p.S741L & vav 2 guanine nucleotide exchange factor \\
\hline BTC-2 & $\mathrm{ZC} 2 \mathrm{HC} 1 \mathrm{~B}$ & 153918 & 6 & 144224199 & 144224199 Missense_Mutation & $\mathrm{C}$ & $\mathrm{T}$ & C. $508 \mathrm{C}>\mathrm{T}$ & c.(508-510 p.P170S & zinc finger, $\mathrm{C} 2 \mathrm{HC}$-type containing $1 \mathrm{~B}$ \\
\hline BTC-2 & ZFP69B & 65243 & 1 & 40928270 & 40928270 Missense_Mutation & $\mathrm{G}$ & $A$ & c. $614 G>A$ & c. $(613-615$ p.G205D & ZFP69 zinc finger protein $B$ \\
\hline BTC-2 & ZNF423 & 23090 & 16 & 49669726 & 49669726 Missense_Mutation & $\mathrm{C}$ & $\mathrm{T}$ & c. $3337 \mathrm{G}>\mathrm{A}$ & c.(3337-33 p.A1113T & zinc finger protein 423 \\
\hline BTC-4 & ARID1A & 8289 & 1 & 27023831 & 27023831 Frame_Shift_Del & G & - & c.937delG & c.(937-939 p.G314fs & AT rich interactive domain $1 \mathrm{~A}$ (SWI-like) \\
\hline BTC-4 & TULP1 & 7287 & 6 & 35480415 & 35480415 Splice_Site & C & G & & c.e $2+1$ & tubby like protein 1 \\
\hline BTC-4 & ACOXL & 55289 & 2 & 111850501 & 111850501 Missense_Mutation & $\mathrm{T}$ & $A$ & c. $1590 \mathrm{~T}>\mathrm{A}$ & c.(1588-15 p.H530Q & acyl-CoA oxidase-like \\
\hline BTC-4 & CACNA1H & 8912 & 16 & 1270626 & 1270626 Missense_Mutation & $\mathrm{C}$ & $A$ & c. $6676 \mathrm{C}>\mathrm{A}$ & c. $(6676-66$ p.L2226M & calcium channel, voltage-dependent, $\mathrm{T}$ type, alpha $1 \mathrm{H}$ subunit \\
\hline BTC-4 & CATSPERB & 79820 & 14 & 92174496 & 92174496 Missense_Mutation & $\mathrm{T}$ & $A$ & c. $455 \mathrm{~A}>\mathrm{T}$ & c.(454-456 p.D152V & catsper channel auxiliary subunit beta \\
\hline BTC-4 & CDKN2A & 1029 & 9 & 21971035 & 21971035 Missense_Mutation & $\mathrm{T}$ & $\mathrm{C}$ & c. $170 A>G$ & c.(169-171 p.D57G & cyclin-dependent kinase inhibitor $2 \mathrm{~A}$ \\
\hline BTC-4 & CES3 & 23491 & 16 & 67006620 & 67006620 Missense_Mutation & $\mathrm{G}$ & A & c. $408 \mathrm{G}>\mathrm{A}$ & c. $(406-408$ p.M136) & carboxylesterase 3 \\
\hline BTC-4 & CLCNKA & 1187 & 1 & 16349137 & 16349137 Missense_Mutation & G & A & c. $23 \mathrm{G}>\mathrm{A}$ & c. $(22-24) c(p . R 8 H$ & chloride channel, voltage-sensitive Ka \\
\hline BTC-4 & COL22A1 & 169044 & 8 & 139890232 & 139890232 Missense_Mutation & C & $\mathrm{T}$ & c. $419 \mathrm{G}>\mathrm{A}$ & c.(418-420 p.R140H & collagen, type XXII, alpha 1 \\
\hline BTC-4 & CRISPLD1 & 83690 & 8 & 75941665 & 75941665 Missense_Mutation & G & $A$ & c. $1364 G>A$ & c.(1363-13 p.R455Q & cysteine-rich secretory protein LCCL domain containing 1 \\
\hline BTC-4 & DAW1 & 164781 & 2 & 228767727 & 228767727 Missense_Mutation & $\mathrm{T}$ & $\mathrm{C}$ & C.550T >C & c.(550-552 p.S184P & dynein assembly factor with WDR repeat domains 1 \\
\hline BTC-4 & DYSF & 8291 & 2 & 71797023 & 71797023 Missense_Mutation & G & $A$ & c. $2938 G>A$ & c.(2938-29. p.G980R & dysferlin \\
\hline BTC-4 & ELN & 2006 & 7 & 73461047 & 73461047 Missense_Mutation & $\mathrm{C}$ & $\mathrm{T}$ & c. $593 C>T$ & c.(592-594 p.P198L & elastin \\
\hline BTC-4 & FAM135B & 51059 & 8 & 139165339 & 139165339 Missense_Mutation & A & G & c.1379T>C & c. $(1378-13$ p.L460P & family with sequence similarity 135 , member $B$ \\
\hline BTC-4 & FAM208B & 54906 & 10 & 5803432 & 5803432 Missense_Mutation & $\mathrm{C}$ & A & c. $7172 \mathrm{C}>\mathrm{A}$ & c.(7171-71 p.T2391K & family with sequence similarity 208 , member $B$ \\
\hline BTC-4 & GPR65 & 8477 & 14 & 88478153 & 88478153 Missense_Mutation & G & $A$ & c. $962 \mathrm{G}>\mathrm{A}$ & c. $(961-963$ p.R321H & G protein-coupled receptor 65 \\
\hline BTC-4 & GUCY1A3 & 2982 & 4 & 156643206 & 156643206 Missense_Mutation & A & $\mathrm{T}$ & c. $1733 \mathrm{~A}>\mathrm{T}$ & c.(1732-17 p.H578L & guanylate cyclase 1 , soluble, alpha 3 \\
\hline BTC-4 & HID1 & 283987 & 17 & 72959068 & 72959068 Missense_Mutation & $\mathrm{T}$ & $A$ & c. $496 \mathrm{~A}>\mathrm{T}$ & c. $(496-498$ p.S166C & HID1 domain containing \\
\hline BTC-4 & HIP1 & 3092 & 7 & 75189111 & 75189111 Missense_Mutation & G & A & c. $1300 C>T$ & c. $(1300-13$ p.R434W & huntingtin interacting protein 1 \\
\hline BTC-4 & IRF3 & 3661 & 19 & 50167939 & 50167939 Missense_Mutation & $\mathrm{T}$ & $\mathrm{C}$ & c. $157 A>G$ & c. $(157-159$ p. $.153 \mathrm{~V}$ & interferon regulatory factor 3 \\
\hline BTC-4 & KCNB1 & 3745 & 20 & 48098906 & 48098906 Missense_Mutation & c & $T$ & c. $112 \mathrm{G}>\mathrm{A}$ & c.(112-114 p.G38R & $\begin{array}{l}\text { potassium voltage-gated channel, Shab-related subfamily, } \\
\text { member } 1\end{array}$ \\
\hline BTC-4 & KCNT1 & 57582 & 9 & 138664603 & 138664603 Missense_Mutation & $\mathrm{C}$ & $\mathrm{T}$ & c. $2051 C>T$ & c.(2050-20 p.T684M & potassium channel, subfamily $T$, member 1 \\
\hline BTC-4 & LLGL1 & 3996 & 17 & 18143990 & 18143990 Missense_Mutation & $\mathrm{C}$ & $\mathrm{T}$ & c. $2305 C>T$ & c.(2305-23 p.R769W & lethal giant larvae homolog 1 (Drosophila) \\
\hline BTC-4 & LPHN1 & 22859 & 19 & 14294398 & 14294398 Missense_Mutation & G & $A$ & c. $17 \mathrm{C}>\mathrm{T}$ & c. $(16-18) g(p . A 6 V$ & latrophilin 1 \\
\hline BTC-4 & MARS & 4141 & 12 & 57906626 & 57906626 Missense_Mutation & A & $\mathrm{T}$ & c. $1846 \mathrm{~A}>\mathrm{T}$ & c. $(1846-18 \cdot p .1616 \mathrm{~F}$ & methionyl-tRNA synthetase \\
\hline BTC-4 & MBTPS1 & 8720 & 16 & 84093019 & 84093019 Missense_Mutation & $\mathrm{G}$ & $A$ & c. $2719 C>T$ & c.(2719-27 p.R907W & membrane-bound transcription factor peptidase, site 1 \\
\hline BTC-4 & PDC & 5132 & 1 & 186413572 & 186413572 Missense_Mutation & $\mathrm{G}$ & $A$ & c. $280 C>T$ & c. $(280-282$ p.R94C & phosducin \\
\hline BTC-4 & PTTG1IP & 754 & 21 & 46285352 & 46285352 Missense_Mutation & C & G & c. $126 \mathrm{G}>\mathrm{C}$ & c.(124-126 p.Q42H & pituitary tumor-transforming 1 interacting protein \\
\hline
\end{tabular}




\begin{tabular}{|c|c|c|c|c|c|}
\hline BTC-4 & RBBP6 & 5930 & 16 & 24581304 & 24581304 Missense_Mutation \\
\hline BTC-4 & RBBP7 & $5931 \times$ & & 16871823 & 16871823 Missense_Mutation \\
\hline BTC-4 & $\mathrm{RDH} 10$ & 157506 & 8 & 74234976 & 74234976 Missense_Mutation \\
\hline BTC-4 & RFX8 & 731220 & 2 & 102018971 & 102018971 Missense_Mutation \\
\hline BTC-4 & SEMA6D & 80031 & 15 & 48058143 & 48058143 Missense_Mutation \\
\hline BTC-4 & SLC6A17 & 388662 & 1 & 110734682 & 110734682 Missense_Mutation \\
\hline BTC-4 & TMEM205 & 374882 & 19 & 11455971 & 11455971 Missense_Mutation \\
\hline BTC-4 & TRIM4 & 89122 & 7 & 99506284 & 99506284 Missense_Mutation \\
\hline BTC-5 & HMCN1 & 83872 & 1 & 186057081 & 186057084 Frame_Shift_Del \\
\hline BTC-5 & UPP2 & 151531 & 2 & 158977957 & 158977958 Frame_Shift_Ins \\
\hline BTC-5 & FNTB & 2342 & 14 & 65511053 & 65511053 Nonsense_Mutation \\
\hline BTC-5 & ACSM2A & 123876 & 16 & 20482871 & 20482871 Nonsense_Mutation \\
\hline BTC-5 & PSD3 & 23362 & 8 & 18430175 & 18430175 Nonsense_Mutation \\
\hline BTC-5 & COL11A1 & 1301 & 1 & 103467527 & 103467527 Splice_Site \\
\hline BTC-5 & AC007405. & 0 & 2 & 171570595 & 171570595 Missense_Mutation \\
\hline BTC-5 & AC009365. & 0 & 7 & 132412557 & 132412557 Missense_Mutation \\
\hline BTC-5 & ADAM21 & 8747 & 14 & 70924614 & 70924614 Missense_Mutation \\
\hline BTC-5 & APOOL & $139322 X$ & & 84329077 & 84329077 Missense_Mutation \\
\hline BTC-5 & ASPM & 259266 & 1 & 197074062 & 197074062 Missense_Mutation \\
\hline BTC-5 & ATP7A & $538 x$ & & 77301051 & 77301051 Missense_Mutation \\
\hline BTC-5 & ATP8B4 & 79895 & 15 & 50288883 & 50288883 Missense_Mutation \\
\hline BTC-5 & CACNA1A & 773 & 19 & 13616812 & 13616812 Missense_Mutation \\
\hline BTC-5 & CCDC175 & 729665 & 14 & 60031860 & 60031860 Missense_Mutation \\
\hline BTC-5 & CCL18 & 6362 & 17 & 34398340 & 34398340 Missense_Mutation \\
\hline BTC-5 & CD163 & 9332 & 12 & 7651580 & 7651580 Missense_Mutation \\
\hline BTC-5 & COL21A1 & 81578 & 6 & 55922544 & 55922544 Missense_Mutation \\
\hline BTC-5 & CSMD3 & 114788 & 8 & 113697935 & 113697935 Missense_Mutation \\
\hline BTC-5 & CXorf28 & $1 \mathrm{E}+08 \mathrm{X}$ & & 3189922 & 3189922 Missense_Mutation \\
\hline BTC-5 & DMXL2 & 23312 & 15 & 51791693 & 51791693 Missense_Mutation \\
\hline BTC-5 & GAS2 & 2620 & 11 & 22747946 & 22747946 Missense_Mutation \\
\hline BTC-5 & GPR98 & 84059 & 5 & 89939702 & 89939702 Missense_Mutation \\
\hline BTC-5 & HOXA4 & 3201 & 7 & 27170310 & 27170310 Missense_Mutation \\
\hline BTC-5 & $\mathrm{HP}$ & 3240 & 16 & 72090131 & 72090131 Missense_Mutation \\
\hline BTC-5 & ICAM5 & 7087 & 19 & 10405259 & 10405259 Missense_Mutation \\
\hline BTC-5 & KIAA1244 & 57221 & 6 & 138576619 & 138576619 Missense_Mutation \\
\hline BTC-5 & KIF21B & 23046 & 1 & 200948693 & 200948693 Missense_Mutation \\
\hline BTC-5 & KRTAP21-1 & 337977 & 21 & 32127554 & 32127554 Missense_Mutation \\
\hline BTC-5 & LCA10 & $0 \times$ & & 153152431 & 153152431 Missense_Mutation \\
\hline
\end{tabular}

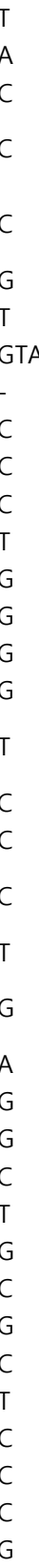

c.3293A $>$ T C.(3292-32 p.E1098V retinoblastoma binding protein 6

c.872A $>$ T c.(871-873 p.D291V retinoblastoma binding protein 7

c.833A $>\mathrm{G}$ c.(832-834 p.K278R retinol dehydrogenase 10 (all-trans)

c.1172G>A c.(1171-11 p.R391Q RFX family member 8, lacking RFX DNA binding domain

c.1505C $>$ T C.(1504-15 p.A502V $\begin{aligned} & \text { sema domain, transmembrane domain (TM), and cytoplasmic } \\ & \text { domain, (semaphorin) } 6 \mathrm{D}\end{aligned}$

c.953C>A C.(952-954 p.A318D $\begin{aligned} & \text { solute carrier family } 6 \text { (neutral amino acid transporter), member } \\ & 17\end{aligned}$

c.221C>T c.(220-222 p.S74L transmembrane protein 205

c.719A $>$ G c. $(718-720$ p.N240S tripartite motif containing 4

c.9381_9384delGT c.(9379-93 p.QY3127f hemicentin 1

c.491_492insT c.(490-495 p.V165fs uridine phosphorylase 2

c.847C $>$ T c. $\quad$ (847-849 p.R283* farnesyltransferase, CAAX box, beta

c.754C $>\mathrm{T} \quad$ c. $(754-756$ p.Q252* acyl-CoA synthetase medium-chain family member $2 \mathrm{~A}$

c.2647G > T c. (2647-26. p.E883* pleckstrin and Sec7 domain containing 3

c.e24-2

collagen, type $\mathrm{XI}$, alpha 1

c. $359 C>\mathrm{G}$

c. $409 \mathrm{G}>\mathrm{A}$

c.398G $>\mathrm{T}$

c. $560 \mathrm{G}>\mathrm{C}$

c.(358-360 p.A120G

c.(409-411 p.V137I

c.(397-399 p.R133L ADAM metallopeptidase domain 21

c.(559-561 p.S187T apolipoprotein O-like

c. $4319 A>G$

c. $4208 \mathrm{C}>\mathrm{A}$

c. $580 \mathrm{G}>\mathrm{C}$

c.(4318-43 p.Q1440R $\begin{aligned} & \text { asp (abnorm } \\ & \text { (Drosophila) }\end{aligned}$

c.(4207-42 p.S1403Y ATPase, Cu++ transporting, alpha polypeptide

c.(580-582 p.G194R ATPase, class I, type 8B, member 4

c. $227 \mathrm{G}>\mathrm{T}$

c. $625 \mathrm{~A}>\mathrm{G}$

c.(226-228 p.R76L calcium channel, voltage-dependent, P/Q type, alpha 1A subunit

c.209G $>A$

c.662T $>C$

c. $2785 \mathrm{C}>\mathrm{A}$

c. $2182 C>G$

c. $49 \mathrm{C}>\mathrm{T}$

c. $3728 \mathrm{~A}>\mathrm{T}$

c.376G $>A$

c. $2636 C>T$

c. $43 \mathrm{C}>\mathrm{A}$

c $77 \mathrm{C}>\mathrm{T}$

c. $2173 \mathrm{~T}>\mathrm{C}$

C. $817 \mathrm{C}>\mathrm{T}$

c. $4130 \mathrm{G}>\mathrm{A}$

c.(625-627 p.1209V coiled-coil domain containing 175

c.(208-210 p.C70Y

chemokine (C-C motif) ligand 18 (pulmonary and activation-

c.(661-663 p.1221T CD163 molecule

c.(2785-27 p.Q929K collagen, type XXI, alpha 1

c.(2182-21 p.P728A CUB and Sushi multiple domains 3

c.(49-51)C(p.P17S chromosome $X$ open reading frame 28

c.(3727-37 p.D1243V Dmx-like 2

c.(376-378 p.D126N growth arrest-specific 2

c.(2635-26 p.T879M G protein-coupled receptor 98

c. (43-45)Cip.P15T homeobox A4

c. (76-78)a(p.T26M haptoglobin

c. (4129-41 p.R1377Q kinesin family member 21B

c. 143G $>T \quad$ c. $(142-144$ p.C48F keratin associated protein 21-1

c.311G $>\mathrm{T} \quad$ c. $(310-312$ p.R104L 


\begin{tabular}{|c|c|c|c|c|c|}
\hline BTC-5 & MAP1B & 4131 & 5 & 71490888 & 71490888 Missense_Mutation \\
\hline BTC-5 & MLK4 & 0 & 1 & 233518335 & 233518335 Missense_Mutation \\
\hline BTC-5 & NBEAL2 & 23218 & 3 & 47041430 & 47041430 Missense_Mutation \\
\hline BTC-5 & NFATC2 & 4773 & 20 & 50140482 & 50140482 Missense_Mutation \\
\hline BTC-5 & NRAS & 4893 & 1 & 115256529 & 115256529 Missense_Mutation \\
\hline BTC-5 & OLFML2B & 25903 & 1 & 161967939 & 161967939 Missense_Mutation \\
\hline BTC-5 & OSBPL5 & 114879 & 11 & 3143583 & 3143583 Missense_Mutation \\
\hline BTC-5 & PCDHB1 & 29930 & 5 & 140432385 & 140432385 Missense_Mutation \\
\hline BTC-5 & PCSK5 & 5125 & 9 & 78936474 & 78936474 Missense_Mutation \\
\hline BTC-5 & PGR & 5241 & 11 & 100998706 & 100998706 Missense_Mutation \\
\hline BTC-5 & PHIP & 55023 & 6 & 79695085 & 79695085 Missense_Mutation \\
\hline BTC-5 & PIK3C2G & 5288 & 12 & 18435656 & 18435656 Missense_Mutation \\
\hline BTC-5 & PRKCD & 5580 & 3 & 53219710 & 53219710 Missense_Mutation \\
\hline BTC-5 & SEC14L2 & 23541 & 22 & 30802345 & 30802345 Missense_Mutation \\
\hline BTC-5 & SEMA6A & 57556 & 5 & 115782872 & 115782872 Missense_Mutation \\
\hline BTC-5 & SKIV2L2 & 23517 & 5 & 54635888 & 54635888 Missense_Mutation \\
\hline BTC-5 & TAF1L & 138474 & 9 & 32631848 & 32631848 Missense_Mutation \\
\hline BTC-5 & TEX15 & 56154 & 8 & 30702546 & 30702546 Missense_Mutation \\
\hline BTC-5 & TLR8 & $51311 x$ & & 12939230 & 12939230 Missense Mutation \\
\hline BTC-5 & TMEM200 & 114801 & 6 & 130762039 & 130762039 Missense_Mutation \\
\hline BTC-5 & TMEM80 & 283232 & 11 & 703112 & 703112 Missense_Mutation \\
\hline BTC-5 & TRPM6 & 140803 & 9 & 77377993 & 77377993 Missense_Mutation \\
\hline BTC-5 & TSPYL5 & 85453 & 8 & 98290039 & 98290039 Missense_Mutation \\
\hline BTC-6 & AMIGO2 & 347902 & 12 & 47471598 & 47471598 Missense_Mutation \\
\hline BTC-6 & BIRC6 & 57448 & 2 & 32712788 & 32712788 Missense_Mutation \\
\hline BTC-6 & BZW1 & 9689 & 2 & 201680210 & 201680210 Missense_Mutation \\
\hline BTC-6 & CD200R1 & 131450 & 3 & 112648303 & 112648303 Missense_Mutation \\
\hline BTC-6 & CRYBG3 & 131544 & 3 & 97593426 & 97593426 Missense_Mutation \\
\hline BTC-6 & DEAF1 & 10522 & 11 & 694992 & 694992 Missense_Mutation \\
\hline BTC-6 & EFCAB5 & 374786 & 17 & 28295933 & 28295933 Missense_Mutation \\
\hline BTC-6 & FSIP2 & 401024 & 2 & 186671383 & 186671383 Missense_Mutation \\
\hline BTC-6 & GAPVD1 & 26130 & 9 & 128064438 & 128064438 Missense_Mutation \\
\hline BTC-6 & IQSEC2 & $23096 \mathrm{X}$ & & 53349985 & 53349985 Missense_Mutation \\
\hline BTC-6 & KDM5A & 5927 & 12 & 427378 & 427378 Missense_Mutation \\
\hline BTC-6 & LPAR3 & 23566 & 1 & 85279695 & 85279695 Missense_Mutation \\
\hline BTC-6 & NLRP1 & 22861 & 17 & 5462767 & 5462767 Missense_Mutation \\
\hline BTC-6 & OR6B3 & 150681 & 2 & 240984973 & 240984973 Missense_Mutation \\
\hline BTC- 6 & OTUD6A & $139562 \times$ & & 69282627 & 69282627 Missense Mutation \\
\hline
\end{tabular}

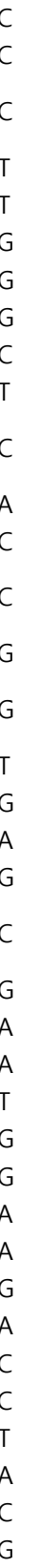

$1706 C>G$

c. $2989 C>T$

c $3841 \mathrm{C}>\mathrm{A}$

c.298G $>A$

c. $182 A>G$

c.1153A>T

c. $295 C>T$

c. $1330 \mathrm{G}>\mathrm{A}$

c.3940G $>A$

c. $1096 \mathrm{G}>\mathrm{A}$

c. $2521 A>C$

c. $641 \mathrm{C}>\mathrm{A}$

c.979A $>\mathrm{T}$

c. $145 \mathrm{C}>\mathrm{G}$

c. $2530 \mathrm{G}>\mathrm{A}$

c.566G $>\mathrm{T}$

c. $3730 \mathrm{C}>\mathrm{T}$

c. $3988 A>C$

c. $2125 G>C$

c. $472 A>G$

c. $469 \mathrm{G}>\mathrm{T}$

c.3594G $>\mathrm{T}$

c. $34 \mathrm{C}>\mathrm{T}$

c. $1188 \mathrm{~T}>\mathrm{G}$

c. $7888 \mathrm{~A}>\mathrm{T}$

c. $211 \mathrm{~T}>\mathrm{G}$

c. $185 C>T$

c. $3388 \mathrm{G}>\mathrm{T}$

c. $56 \mathrm{~T}>\mathrm{C}$

c. $315 \mathrm{~A}>\mathrm{T}$

c. $17350 \mathrm{G}>\mathrm{A}$

c. $362 A>T$

c. $337 \mathrm{G}>\mathrm{A}$

c. $2791 \mathrm{G}>\mathrm{A}$

c. $896 \mathrm{~A}>\mathrm{G}$

c. 1249T >C

C. $517 \mathrm{G}>\mathrm{T}$

c. $253 \mathrm{G}>\mathrm{C}$ c.(1705-17 p.T569R microtubule-associated protein 1B

c.(2989-29 p.P997S

c.(3841-38.p.Q1281K neurobeachin-like 2

c.(298-300 p.A100T nuclear factor of activated T-cells, cytoplasmic, calcineurindependent 2

c.(181-183 p.Q61R neuroblastoma RAS viral (v-ras) oncogene homolog

c.(1153-11 p.S385C olfactomedin-like $2 B$

c.(295-297 p.L99F oxysterol binding protein-like 5

c.(1330-13 p.D444N protocadherin beta 1

c.(3940-39. p.E1314K proprotein convertase subtilisin/kexin type 5

c.(1096-10 p.D366N progesterone receptor

c.(2521-25 p.S841R pleckstrin homology domain interacting protein

c.(640-642 p.P214H phosphatidylinositol-4-phosphate 3-kinase, catalytic subunit type 2 gamma

c.(979-981 p.M327L protein kinase $C$, delta

c.(145-147 p.L49V SEC14-like 2 (S. cerevisiae)

c.(2530-25 p.E844K sema domain, transmembrane domain (TM), and cytoplasmic domain, (semaphorin) $6 \mathrm{~A}$

c.(565-567 p.S189| superkiller viralicidic activity 2-like 2 (S. cerevisiae)

c.(3730-37 p.R1244W TAF1 RNA polymerase II, TATA box binding protein (TBP)associated factor, $210 \mathrm{kDa}$-like

c.(3988-39 p.K1330Q testis expressed 15

c.(2125-21 p.E709Q toll-like receptor 8

c.(472-474 p.1158V transmembrane protein 200A

c.(469-471 p.V157F transmembrane protein 80

c.(3592-35 p.K1198N transient receptor potential cation channel, subfamily M, member

c. $3592-35$ p.K1198N 6

c.(34-36)Cıp.R12C TSPY-like 5

c.(1186-11 p.F396L adhesion molecule with Ig-like domain 2

c.(7888-78 p.I2630F baculoviral IAP repeat containing 6

c.(211-213 p.F71V basic leucine zipper and W2 domains 1

c.(184-186 p.P62L CD200 receptor 1

c.(3388-33 p.G1130W beta-gamma crystallin domain containing 3

c.(55-57)g p.V19A DEAF1 transcription factor

c.(313-315 p.E105D EF-hand calcium binding domain 5

c.(17350-1 p.A5784T fibrous sheath interacting protein 2

c.(361-363 p.N121। GTPase activating protein and VPS9 domains 1

c.337-339 p.D113N IQ motif and Sec7 domain 2

c.(2791-27 p.G931R lysine (K)-specific demethylase 5A

c.(895-897 p.D299G lysophosphatidic acid receptor 3

c.(1249-12 p.W417R NLR family, pyrin domain containing

c.(517-519 p.V173F olfactory receptor, family 6 , subfamily B, member 3

c.(253-255 p.E85Q OTU deubiquitinase 6A 


\begin{tabular}{|c|c|c|c|c|c|}
\hline BTC-6 & PTPRH & 5794 & 19 & 55715264 & 55715264 Missense_Mutation \\
\hline BTC-6 & RAD50 & 10111 & 5 & 131927660 & 131927660 Missense_Mutation \\
\hline BTC-6 & RB1 & 5925 & 13 & 49039417 & 49039417 Missense_Mutation \\
\hline BTC-6 & SAMD9L & 219285 & 7 & 92763376 & 92763376 Missense_Mutation \\
\hline BTC-6 & SERINC5 & 256987 & 5 & 79454696 & 79454696 Missense_Mutation \\
\hline BTC-6 & ST6GALNA & 81849 & 1 & 77515950 & 77515950 Missense_Mutation \\
\hline BTC-6 & TMEM47 & $83604 x$ & & 34657486 & 34657486 Missense_Mutation \\
\hline BTC-6 & TRIM41 & 90933 & 5 & 180651595 & 180651595 Missense_Mutation \\
\hline BTC-6 & WDSUB1 & 151525 & 2 & 160139525 & 160139525 Missense_Mutation \\
\hline BTC-6 & ZC3H13 & 23091 & 13 & 46616352 & 46616352 Missense_Mutation \\
\hline BTC-7 & CCDC18 & 343099 & 1 & 93705341 & 93705341 Nonsense_Mutation \\
\hline BTC-7 & KANSL1 & 284058 & 17 & 44115975 & 44115975 Nonsense_Mutation \\
\hline BTC-7 & PLEKHG2 & 64857 & 19 & 39912773 & 39912773 Nonsense_Mutation \\
\hline BTC-7 & ZNF546 & 339327 & 19 & 40520465 & 40520465 Nonsense_Mutation \\
\hline BTC-7 & SI & 6476 & 3 & 164760855 & 164760855 Nonsense_Mutation \\
\hline BTC-7 & SLITRK2 & $84631 x$ & & 144904042 & 144904042 Nonsense_Mutation \\
\hline BTC-7 & CNTNAP5 & 129684 & 2 & 125367471 & 125367471 Missense_Mutation \\
\hline BTC-7 & DNMT3A & 1788 & 2 & 25467461 & 25467461 Missense_Mutation \\
\hline BTC-7 & DSG4 & 147409 & 18 & 28971077 & 28971077 Missense_Mutation \\
\hline BTC-7 & EPC1 & 80314 & 10 & 32560813 & 32560813 Missense_Mutation \\
\hline BTC-7 & ESM1 & 11082 & 5 & 54277867 & 54277867 Missense_Mutation \\
\hline BTC-7 & F13A1 & 2162 & 6 & 6266956 & 6266956 Missense_Mutation \\
\hline BTC-7 & FCRL3 & 115352 & 1 & 157665154 & 157665154 Missense_Mutation \\
\hline BTC-7 & FUT1 & 2523 & 19 & 49254093 & 49254093 Missense_Mutation \\
\hline BTC-7 & GLI3 & 2737 & 7 & 42005951 & 42005951 Missense_Mutation \\
\hline BTC-7 & HEATR1 & 55127 & 1 & 236721708 & 236721708 Missense_Mutation \\
\hline BTC-7 & KLHL24 & 54800 & 3 & 183368493 & 183368493 Missense_Mutation \\
\hline BTC-7 & KLHL9 & 55958 & 9 & 21333179 & 21333179 Missense_Mutation \\
\hline BTC-7 & КМT2A & 4297 & 11 & 118392628 & 118392628 Missense_Mutation \\
\hline BTC-7 & KRAS & 3845 & 12 & 25398284 & 25398284 Missense_Mutation \\
\hline BTC-7 & LPP & 4026 & 3 & 188326976 & 188326976 Missense_Mutation \\
\hline BTC-7 & LRP1B & 53353 & 2 & 141707920 & 141707920 Missense_Mutation \\
\hline BTC-7 & MSH3 & 4437 & 5 & 79950739 & 79950739 Missense_Mutation \\
\hline BTC-7 & NCOR1 & 9611 & 17 & 16049747 & 16049747 Missense_Mutation \\
\hline BTC-7 & NIPBL & 25836 & 5 & 37064917 & 37064917 Missense_Mutation \\
\hline BTC-7 & NTRK1 & 4914 & 1 & 156851340 & 156851340 Missense_Mutation \\
\hline BTC-7 & PELP1 & 27043 & 17 & 4575146 & 4575146 Missense_Mutation \\
\hline BTC-7 & RELN & 5649 & 7 & 103183183 & 103183183 Missense_Mutation \\
\hline
\end{tabular}




\begin{tabular}{|c|c|c|c|c|c|c|c|c|c|c|}
\hline BTC-7 & SPTBN1 & 6711 & 2 & 54871578 & 54871578 Missense_Mutation & G & $A$ & c. $4124 \mathrm{G}>\mathrm{A}$ & c.(4123-41 p.R1375Q & spectrin, beta, non-erythrocytic 1 \\
\hline BTC-7 & SUSD2 & 56241 & 22 & 24583685 & 24583685 Missense_Mutation & G & $\mathrm{T}$ & $c .2038 G>T$ & c.(2038-20.p.A680S & sushi domain containing 2 \\
\hline BTC-7 & ZNF14 & 7561 & 19 & 19822422 & 19822422 Missense_Mutation & C & A & c.1668G>T & c.(1666-16 p.E556D & zinc finger protein 14 \\
\hline BTC-8 & APC & 324 & 5 & 112175952 & 112175952 Frame_Shift_Del & A & - & c.4661 delA & c. $(4660-46$ p.E1554fs & adenomatous polyposis coli \\
\hline BTC-8 & LMO7 & 4008 & 13 & 76408475 & 76408475 Nonsense_Mutation & C & $\mathrm{T}$ & c. $2479 C>T$ & c. $(2479-24$ p.Q827* & LIM domain 7 \\
\hline BTC-8 & TP53 & 7157 & 17 & 7574003 & 7574003 Nonsense_Mutation & G & A & c. $1024 C>T$ & c.(1024-10 p.R342* & tumor protein p53 \\
\hline BTC-8 & DUXA & 503835 & 19 & 57670637 & 57670637 Nonsense_Mutation & G & $A$ & c. $190 C>T$ & c.(190-192 p.Q64* & double homeobox A \\
\hline BTC-8 & MMS22L & 253714 & 6 & 97676947 & 97676947 Nonsense_Mutation & $C$ & $\mathrm{~T}$ & c. $1862 \mathrm{G}>\mathrm{A}$ & c.(1861-18 p.W621* & MMS22-like, DNA repair protein \\
\hline BTC-8 & KIF15 & 56992 & 3 & 44843467 & 44843467 Splice_Site & G & C & & c.e13+1 & kinesin family member 15 \\
\hline BTC-8 & ABCA9 & 10350 & 17 & 67025361 & 67025361 Missense_Mutation & $\mathrm{T}$ & G & c. $1453 \mathrm{~A}>\mathrm{C}$ & c. $(1453-14$ p.N485H & ATP-binding cassette, sub-family A (ABC1), member 9 \\
\hline BTC-8 & ACSS1 & 84532 & 20 & 25004233 & 25004233 Missense_Mutation & C & $\mathrm{T}$ & c. $676 \mathrm{G}>\mathrm{A}$ & c. $(676-678$ p.G226R & acyl-CoA synthetase short-chain family member 1 \\
\hline BTC-8 & ADAMTS6 & 11174 & 5 & 64595872 & 64595872 Missense_Mutation & G & A & c. $1310 C>T$ & c.(1309-13 p.A437V & ADAM metallopeptidase with thrombospondin type 1 motif, 6 \\
\hline BTC-8 & ADORA1 & 134 & 1 & 203098180 & 203098180 Missense_Mutation & A & G & c. $211 A>G$ & c. $(211-213$ p. $171 \mathrm{~V}$ & adenosine $\mathrm{A} 1$ receptor \\
\hline BTC-8 & $\mathrm{AOC} 3$ & 8639 & 17 & 41006705 & 41006705 Missense_Mutation & C & $\mathrm{T}$ & c. $1841 C>T$ & c.(1840-18 p.P614L & amine oxidase, copper containing 3 \\
\hline BTC-8 & $\mathrm{CPZ}$ & 8532 & 4 & 8603105 & 8603105 Missense_Mutation & G & A & c. $344 G>A$ & c.(343-345 p.R115Q & carboxypeptidase Z \\
\hline BTC-8 & DACT3 & 147906 & 19 & 47151783 & 47151783 Missense_Mutation & G & C & c. $1846 C>G$ & c.(1846-18 p.L616V & dishevelled-binding antagonist of beta-catenin 3 \\
\hline BTC-8 & ENPP2 & 5168 & 8 & 120577135 & 120577135 Missense_Mutation & A & C & c.1097T>G & c. (1096-10 p.V366G & ectonucleotide pyrophosphatase/phosphodiesterase 2 \\
\hline BTC-8 & INO80D & 54891 & 2 & 206870153 & 206870153 Missense_Mutation & C & A & c.2023G>T & c. $(2023-20$ p.A675S & INO80 complex subunit D \\
\hline BTC-8 & IRX3 & 79191 & 16 & 54319378 & 54319378 Missense_Mutation & $\mathrm{T}$ & C & c. $415 A>G$ & c.(415-417 p.T139A & iroquois homeobox 3 \\
\hline BTC-8 & KRAS & 3845 & 12 & 25398281 & 25398281 Missense_Mutation & $\mathrm{C}$ & $\mathrm{T}$ & c.38G $>\mathrm{A}$ & c.(37-39)g(p.G13D & Kirsten rat sarcoma viral oncogene homolog \\
\hline BTC-8 & KRT73 & 319101 & 12 & 53004589 & 53004589 Missense_Mutation & C & $\mathrm{T}$ & c. $1141 \mathrm{G}>\mathrm{A}$ & c.(1141-11.p.A381T & keratin 73 \\
\hline BTC-8 & LAX1 & 54900 & 1 & 203743063 & 203743063 Missense_Mutation & G & A & c. $451 \mathrm{G}>\mathrm{A}$ & c.(451-453 p.A151T & lymphocyte transmembrane adaptor 1 \\
\hline BTC-8 & MATN4 & 8785 & 20 & 43927089 & 43927089 Missense_Mutation & G & A & c. $1147 C>T$ & c.(1147-11.p.R383W & matrilin 4 \\
\hline BTC-8 & NAB1 & 4664 & 2 & 191524551 & 191524551 Missense_Mutation & G & A & c. $649 \mathrm{G}>\mathrm{A}$ & c.(649-651 p.E217K & NGFI-A binding protein 1 (EGR1 binding protein 1) \\
\hline BTC-8 & NPR3 & 4883 & 5 & 32712527 & 32712527 Missense_Mutation & C & A & c. $645 C>A$ & c. (643-645 p.F215L & natriuretic peptide receptor 3 \\
\hline BTC-8 & OTOG & 340990 & 11 & 17655819 & 17655819 Missense_Mutation & A & G & c.7288A>G & c.(7288-72 p.T2430A & otogelin \\
\hline BTC-8 & PCDHGB6 & 56100 & 5 & 140788665 & 140788665 Missense_Mutation & G & A & c. $896 \mathrm{G}>\mathrm{A}$ & c.(895-897 p.G299D & protocadherin gamma subfamily $B, 6$ \\
\hline BTC-8 & POLA1 & $5422 x$ & & 24906154 & 24906154 Missense_Mutation & G & A & c. $4061 \mathrm{G}>\mathrm{A}$ & c. $(4060-40$ p.R1354H & polymerase (DNA directed), alpha 1, catalytic subunit \\
\hline BTC-8 & RALGAPA1 & 253959 & 14 & 36096402 & 36096402 Missense_Mutation & $\mathrm{T}$ & A & c. $5272 A>T$ & c.(5272-52 p.M1758L & Ral GTPase activating protein, alpha subunit 1 (catalytic) \\
\hline BTC-8 & RIPK2 & 8767 & 8 & 90792331 & 90792331 Missense_Mutation & G & $\mathrm{C}$ & c. $882 \mathrm{G}>\mathrm{C}$ & c. (880-882 p.L294F & receptor-interacting serine-threonine kinase 2 \\
\hline BTC-8 & ROBO1 & 6091 & 3 & 78710287 & 78710287 Missense_Mutation & A & G & c. $2213 T>C$ & c.(2212-22 p.1738T & roundabout, axon guidance receptor, homolog 1 (Drosophila) \\
\hline BTC-8 & $\mathrm{ROBO} 2$ & 6092 & 3 & 77657029 & 77657029 Missense_Mutation & C & A & c.3217C>A & c.(3217-32 p.P1073T & roundabout, axon guidance receptor, homolog 2 (Drosophila) \\
\hline BTC-8 & SHISA8 & 440829 & 22 & 42310285 & 42310285 Missense_Mutation & C & A & c. $286 G>T$ & c. (286-288 p.A96S & shisa family member 8 \\
\hline BTC-8 & SMARCA2 & 6595 & 9 & 2104152 & 2104152 Missense_Mutation & $\mathrm{T}$ & c & c.3275T>C & c.(3274-32 p.L1092P & $\begin{array}{l}\text { SWI/SNF related, matrix associated, actin dependent regulator of } \\
\text { chromatin, subfamily a, member } 2\end{array}$ \\
\hline BTC-8 & SSTR1 & 6751 & 14 & 38678956 & 38678956 Missense_Mutation & G & A & c. $362 \mathrm{G}>\mathrm{A}$ & c. $(361-363$ p.R121H & somatostatin receptor 1 \\
\hline BTC-8 & SYN3 & 8224 & 22 & 33265027 & 33265027 Missense_Mutation & C & $\mathrm{T}$ & c. $547 \mathrm{G}>\mathrm{A}$ & c.(547-549 p.G183S & synapsin III \\
\hline BTC-8 & TROVE2 & 6738 & 1 & 193046115 & 193046115 Missense_Mutation & $\mathrm{C}$ & G & c.1021C>G & c.(1021-10 p.L341V & TROVE domain family, member 2 \\
\hline BTC-8 & USH2A & 7399 & 1 & 215844423 & 215844423 Missense_Mutation & C & $\mathrm{T}$ & c. $14024 \mathrm{G}>\mathrm{A}$ & c.(14023-1. p.R4675K & Usher syndrome $2 \mathrm{~A}$ (autosomal recessive, mild) \\
\hline BTC-8 & USH2A & 7399 & 1 & 216062042 & 216062042 Missense_Mutation & G & $\mathrm{C}$ & c. $7949 C>G$ & c.(7948-79 p.P2650R & Usher syndrome 2A (autosomal recessive, mild) \\
\hline BTC-8 & XCR1 & 2829 & 3 & 46062623 & 46062623 Missense_Mutation & G & A & c. $817 C>T$ & c. $(817-819$ p.R273C & chemokine ( $\mathrm{C}$ motif) receptor 1 \\
\hline BTC-8 & ZSCAN20 & 7579 & 1 & 33960845 & 33960845 Missense_Mutation & $\mathrm{C}$ & A & c. $2901 C>A$ & c.(2899-29 p.F967L & zinc finger and SCAN domain containing 20 \\
\hline
\end{tabular}

